\title{
Biomechanics of Human Fetal Hearts with Critical Aortic Stenosis
}

\author{
Chi Wei Ong, ${ }^{1}$ Meifeng Ren, ${ }^{1}$ Hadi Wiputra, ${ }^{1}$ Joy Mojumder, ${ }^{2}$ \\ Wei Xuan Chan, ${ }^{1}$ Andreas Tulzer, ${ }^{3}$ Gerald Tulzer, ${ }^{3}$ \\ Martin Lindsay Buist, ${ }^{1}$ Citra Nurfarah Zaini Mattar, ${ }^{4}$ \\ Lik Chuan Lee, ${ }^{2}$ and Choon Hwai Yap (iD ${ }^{5}$
}

${ }^{1}$ Department of Biomedical Engineering, National University of Singapore, Singapore, Singapore; ${ }^{2}$ Department of Mechanical Engineering, Michigan State University, East Lansing, United States; ${ }^{3}$ Department of Pediatric Cardiology, Children's Heart Center Linz, Kepler University Hospital, Linz, Austria; ${ }^{4}$ Department of Obstetrics and Gynecology, Yong Loo Lin School of Medicine, National University of Singapore, National University Health System, Singapore, Singapore; and ${ }^{5}$ Department of Bioengineering, Imperial College London, London, UK

(Received 23 July 2020; accepted 26 October 2020; published online 11 November 2020)

Associate Editor Lakshmi Prasad Dasi oversaw the review of this article.

\begin{abstract}
Critical aortic stenosis (AS) of the fetal heart causes a drastic change in the cardiac biomechanical environment. Consequently, a substantial proportion of such cases will lead to a single-ventricular birth outcome. However, the biomechanics of the disease is not well understood. To address this, we performed Finite Element (FE) modelling of the healthy fetal left ventricle (LV) based on patientspecific 4D ultrasound imaging, and simulated various disease features observed in clinical fetal AS to understand their biomechanical impact. These features included aortic stenosis, mitral regurgitation (MR) and LV hypertrophy, reduced contractility, and increased myocardial stiffness. AS was found to elevate LV pressures and myocardial stresses, and depending on severity, can drastically decrease stroke volume and myocardial strains. These effects are moderated by MR. AS alone did not lead to MR velocities above $3 \mathrm{~m} / \mathrm{s}$ unless LV hypertrophy was included, suggesting that hypertrophy may be involved in clinical cases with high MR velocities. LV hypertrophy substantially elevated LV pressure, valve flow velocities and stroke volume, while reducing LV contractility resulted in diminished LV pressure, stroke volume and wall strains. Typical extent of hypertrophy during fetal AS in the clinic, however, led to excessive LV pressure and valve velocity in the FE model, suggesting that reduced contractility is typically associated with hypertrophy. Increased LV passive stiffness, which might represent fibroelastosis, was found to have minimal impact on LV pressures, stroke volume, and wall strain. This suggested that fibroelastosis could be a by-product of the disease progression and does not significantly impede cardiac function. Our study demonstrates that FE modelling is a valuable tool for elucidating the biomechanics of congenital heart disease and
\end{abstract}

Address correspondence to Choon Hwai Yap, Department of Bioengineering, Imperial College London, London, UK. Electronic mail: c.yap@imperial.ac.uk can calculate parameters which are difficult to measure, such as intraventricular pressure and myocardial stresses.

Keywords-Fetal aortic stenosis, Evolving hypoplastic left heart syndrome, Fetal mitral regurgitation, Fetal left ventricle, Fetal heart biomechanics, Finite element method.

\section{INTRODUCTION}

Congenital aortic stenosis (AS) carries a prevalence of $0.2-0.5$ per 1000 live births. ${ }^{14}$ It can develop during mid-gestation leading to severe cardiac dysfunction, and a substantial proportion of affected fetuses will develop hypoplastic left heart syndrome by birth. ${ }^{30}$ Aortic stenosis causes left ventricular outflow track obstruction and elevated left ventricular (LV) pressures, ${ }^{9}$ which in turn elevates myocardial stresses. Mitral regurgitation (MR) is also frequently present. ${ }^{111}$ LV outflow obstruction decreases stroke volume and the extent of myocardial strain, and can do so severely. $^{24}$ The LV may develop hypertrophy ${ }^{31}$ and endocardial fibroelastosis. ${ }^{32}$ In critical AS, fetal aortic valvuloplasty can be used as an early intrauterine intervention to relieve the outflow obstruction, with recent studies reporting high technical success rates of up to $94 \%{ }^{16}$ with a $59-73 \%$ likelihood of biventricular function at birth. ${ }^{16,17}$ This is a significant improvement from the $28 \%$ probability of biventricular function at birth without intervention. ${ }^{20}$ 
Both the disease physiology and fetal heart intervention significantly alter the biomechanical environment of the fetal heart, which may influence its subsequent development. Studies in animal models have demonstrated that biomechanical aberrations can lead to cardiac maldevelopment and congenital heart malformations. ${ }^{10,43}$ The success with which minimallyinvasive interventions improved structural heart development corroborated this idea, since such interventions are mechanical in nature. It is thus important to understand the biomechanics of fetal cardiac development and congenital heart malformations, such as critical AS, as this may improve our ability to predict gestational and perinatal outcomes.

To date, however, there have been few studies on the biomechanics of the fetal heart. Dewan et al. developed a finite element strain-based growth model for the fetal heart to test the hypothesis that restrictive mitral inflow could cause Hypoplastic Left Heart Syndrome (HLHS). ${ }^{7}$ Wiputra et al. performed computational modelling to delineate the fluid mechanics of structurally normal and abnormal fetal hearts with Tetralogy of Fallot. ${ }^{51}$ However, our understanding of the biomechanical environment of the fetal heart remains lacking. We address these knowledge gaps in the current study by performing image-based FE computational modelling of the biomechanics of the human fetal heart to understand the individual biomechanical effects of the various components of critical AS,

\section{METHODS}

Our strategy was to first develop FE models of the fetal LV myocardium based on clinical images of healthy fetal hearts, and subsequently modify these models to include various features of critical AS. Features investigated include AS, MR, LV hypertrophy, myocardial fibrosis, and cardiomyopathy.

\section{Data Acquisition and Processing}

4D ultrasound images and valve Doppler velocities were acquired from cardiac imaging of three healthy fetuses at 22, 28 and 32 gestational weeks at the National University Hospital, Singapore. Procedures were approved by the Domain Specific Review Board under protocol 2014/00056, and informed consent was obtained from all participants. Retrospective Doppler velocity data and $2 \mathrm{D}$ B-mode images for an additional 32 fetal hearts with critical AS were acquired from the Kepler University Hospital (KUH), Austria. Procedures were approved under the KUH Institutional Review Board protocol \#1009/2017.
4D B-mode images were acquired in the SpatioTemporal Image Correlation (STIC) mode. Next, semi-automatic segmentation of the myocardium was performed with a custom-written lazy-snapping algorithm and vascular modelling toolkit (VMTK) software at a time point slightly before the end diastolic phase. The segmentation surface was then smoothed with Geomagic Studio ${ }^{\circledR}$ (Geomagic Inc., Morrisville, $\mathrm{NC}$, USA), as previously described. ${ }^{52}$ A validated cardiac motion estimation algorithm was then applied to propagate the segmentation to other time points. ${ }^{50}$ This algorithm involved iterative curve-fitting of a global motion model (spatial B-splines of temporal Fourier function) onto a group of pair-wise image registration at various time points, and was validated via published echo data with MRI ground truths. Figure 1 and supplementary video 1 demonstrate proper tracking of myocardial wall motion.

\section{Mechanical Model of the Fetal Left Ventricle}

The mechanical model and FE modelling of the fetal LV was adapted from previous work, ${ }^{46}$ where further details can be found. The FE model is explained briefly below and in the supplementary text. LV stress was decomposed into active and passive stress tensors. The passive stress tensor was modeled using a Fung-type transversely isotropic, hyperelastic constitutive model $^{21}$ with the strain energy function, $W$, given by

$$
W=\frac{1}{2} C\left(e^{Q}-1\right),
$$

where $C$ is defined as passive stiffness coefficient with

$$
\begin{aligned}
Q= & b_{f f} E_{f f}^{2}+b_{x x}\left(E_{s s}^{2}+E_{n n}^{2}+E_{s n}^{2}+E_{n s}^{2}\right) \\
& +b_{f x}\left(E_{f n}^{2}+E_{n f}^{2}+E_{f s}^{2}+E_{s f}^{2}\right)
\end{aligned}
$$

In Eq. (2), $E_{i j}$ with $(i, j) \epsilon(f, s, n)$ are components of the Green-Lagrange strain tensor $\boldsymbol{E}$, with $f, s$, and $n$ denoting the myocardial fiber, sheet and sheet normal directions, respectively.

Past studies had derived varied conclusions about the passive stiffness of the fetal ventricular myocardium. McPherson et al. ${ }^{33}$ concluded that there was no difference in stiffness between human fetal and adult myocardium, Friedman et al. ${ }^{15}$ found the fetal lamb myocardium to be stiffer than in the adult sheep, and Lahmers et al. ${ }^{27}$ reported similar findings in the porcine myocardium. We took a moderate position in light of this variability and assumed that fetal and adult myocardium had the same passive stiffness. Correspondingly, we adopted previously published adult myocardium passive properties ${ }^{46}$ for our fetal heart models. 

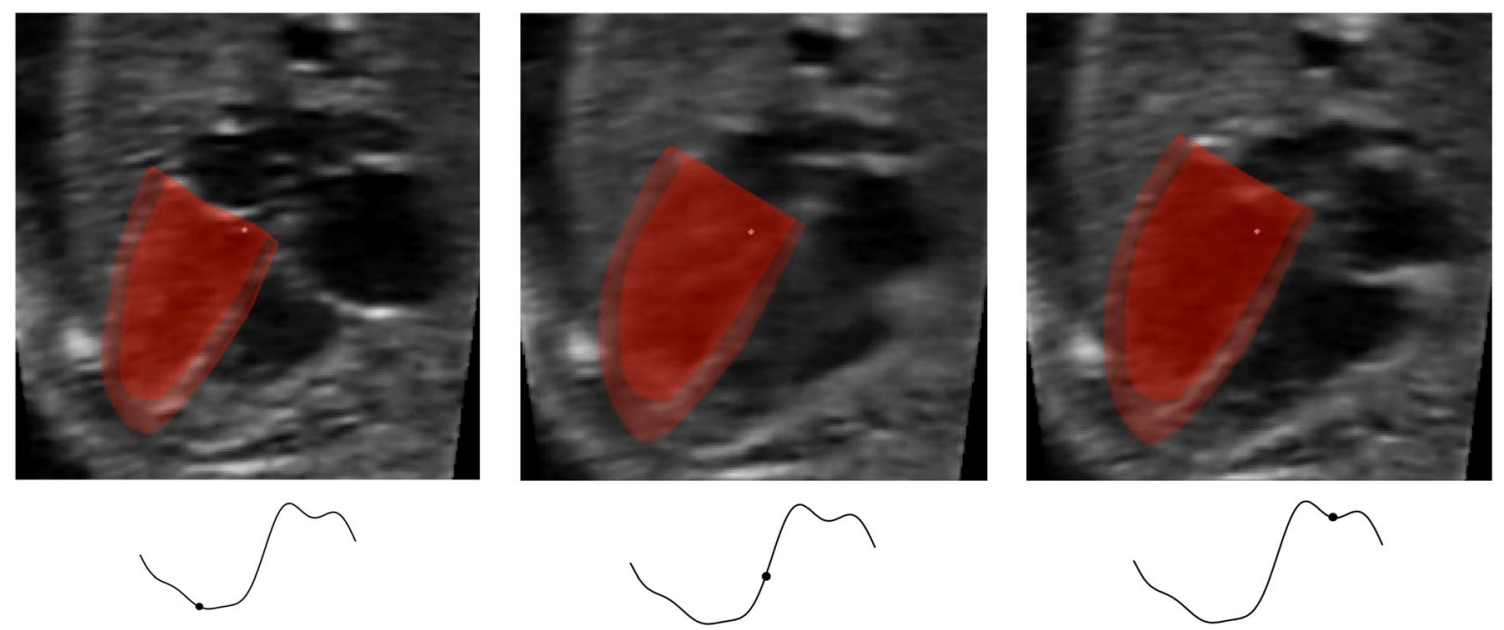

$\mathrm{t}=0.3 \mathrm{~T}$

$\mathrm{t}=0.6 \mathrm{~T}$

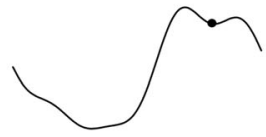

$\mathrm{t}=0.8 \mathrm{~T}$

FIGURE 1. Three-dimensional fetal LV reconstructed from 4D ultrasound for a fetal heart at 22 weeks, with its motion tracked over the cardiac cycle, Here, $t$ denotes time, while $\mathrm{T}$ denotes cardiac cycle duration.

The active stress, $P_{\text {act }}$, was prescribed to act in the local fiber direction using a published active contraction model, 22

$$
P_{\mathrm{act}}=T_{\max } \frac{C a_{0}^{2}}{C a_{0}^{2}+E C a_{50}^{2}} C_{t} e_{f} \otimes e_{f_{0}}
$$

where, $e_{f}$ and $e_{f 0}$ are, respectively, the local vectors defining the muscle fiber direction in the current and reference configurations, $T_{\max }$ is the isometric tension as a result of the longest sarcomere length, and $C a_{0}$ denotes the peak intracellular calcium concentration. $E C a_{50}$ is the length-dependent calcium sensitivity variable, and $C_{t}$ is a time-dependent variable.

Peak myocyte active tension, $T_{\max }$, was assumed to be $60 \mathrm{kPa}$, as an appropriate interpolation between early fetal and adult data. Racca et al. measured fetal myocardium active tension and found that fetal hearts at 130 days and 134 days could generate $49.9 \pm 9.3 \mathrm{kPa}$ and $36.0 \pm 12.1 \mathrm{kPa}$, respectively. ${ }^{42}$ They found that adult hearts generated $75.2 \pm 10.6 \mathrm{kPa}$, while Piroddi et al. measured this to be $108 \pm 7 \mathrm{kPa} .{ }^{41}$ We thus believed that $60 \mathrm{kPa}$ to be an appropriate estimate for $T_{\max }$ in our fetal heart models of all ages. This was corroborated by difficulty in matching the target stroke volume and pressures in our simulations of the 32 week old fetal heart when we had assumed a higher peak active tension of $80 \mathrm{kPa}$ or $100 \mathrm{kPa}$. We did not assume a variability of $T_{\max }$ with gestational age due to the paucity of data. This simplified assumption is a limitation of the study.

Further details and values of parameters used are given in section $\mathrm{S} 1$ of the supplementary text.

\section{Finite Element Model Development and Calibration}

FE modelling was performed using the open source library FEniCS ${ }^{28}$ (https://fenicsproject.org/). Segmented and smoothed LV models were discretized using quadratic tetrahedral elements, with at least 2500 elements, which was higher than past published work, ${ }^{45}$ and which passed our mesh convergence test of less than $2 \%$ change in the resulting stroke volume for a denser mesh size. The fetal LV base was constrained from moving out of the plane and the epicardial edge of the base was fixed. ${ }^{49}$ The myofiber helix angle was assumed to vary with a linear transmural variation from the epidcardium to the endocardium in the LV wall. A simplified Windkessel model, as shown in Fig. 2, was adopted to model the resistances of flow into and out of the LV but was not intended to model other details of the fetal circulation. The initial LV geometry was taken slightly before end-diastole. FE simulation was first performed to increase ventricular pressure to the end diastolic state, and then simulations were conducted for at least 10 additional cardiac cycles before results were analyzed. This was necessary to achieve a steady time-periodic pressure and volume waveforms.

During FE model development and calibration, we attempted to achieve models with reasonable outputs that matched characteristics obtained from the ultrasound images and from available literature. Peak LV systolic pressures were compared to literature values, ventricular volumes were compared to LV geometries obtained from the ultrasound images. The myofiber angle model and the Windkessel model were manually 


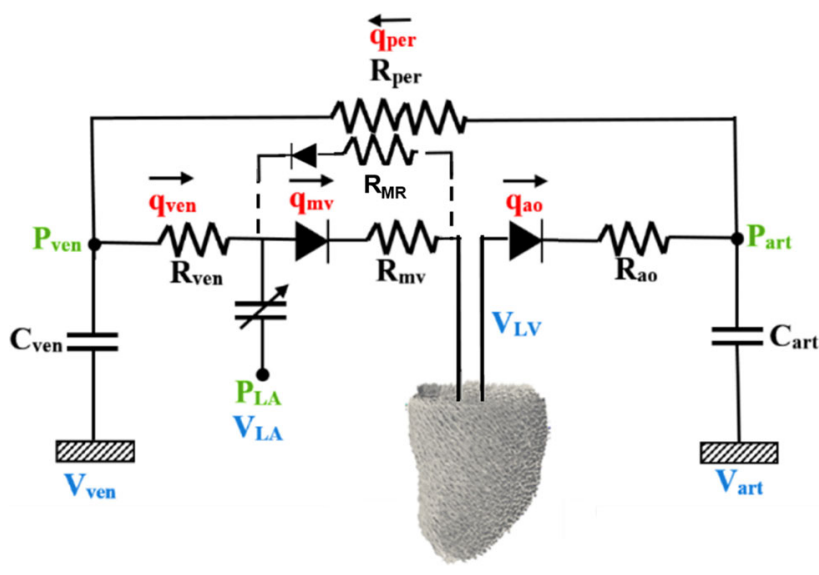

FIGURE 2. Windkessel model employed in the simulations. Elements on the dotted line were not used in healthy scenario modelling, but were added during disease modelling to simulate MR. C is capacitance, $R$ is resistance,

adjusted until a satisfactory match was achieved. This volume comparison was conducted at end-systole, when LV deformations were at their maximum difference from the end-diastolic starting geometry. The starting geometry was propagated to end-systolic state via ultrasound image tracking as described above and via FE simulations, and the resulting end-systolic geometry from both approaches were compared. Evaluation of the geometry match was conducted with the Iterative Closest Point algorithm in Geomagic Studio ${ }^{\circledR},{ }^{26}$ with 15000 random surface points and a tolerance of 0.001 .

Healthy fetal LV pressure data were adopted from Johnson et al. $^{25}$ who invasively measured this in human fetal hearts at gestational ages from 16 to 29 weeks, and provided an empirical linear equation for pressures versus age. We adopted this despite the need for extrapolation for our 32 weeks old fetus due to the lack of such data elsewhere in the literature.

The epi-to-endo myofiber angle that provided the best geometry match with ultrasound images were $-60^{\circ}$ to $60^{\circ}$ for the 32 weeks old fetal heart, $-37^{\circ}$ to $80^{\circ}$ for the 28 weeks old heart, and $-37^{\circ}$ to $83^{\circ}$ for the 22 weeks old heart, where $0^{\circ}$ denoted alignment to the circumferential axis, while $90^{\circ}$ denoted alignment to the apical-basal axis. Our endo-to-epi angles were close to $120^{\circ}$. These values were similar to literature values. Garcia-Canadilla et al. measured endo-to-epi angles of $-50^{\circ}$ to $100^{\circ}$ for mid-2nd trimester human fetus. ${ }^{18}$ Ohayon et al. measured approximately $-60^{\circ}$ to $60^{\circ}$ for 14,20 and 33 weeks old fetal hearts, ${ }^{40}$ while Mekkaoui et al. reported no established fiber orientation for human fetal heart at 10 weeks, but by
19 weeks, there was a $120^{\circ}$ endo-to-epi angle difference. $^{34}$

The Windkessel model parameters that enabled the good match are given in supplementary Table S2 and S3. The heart rate used in 22,28 , and 32 weeks old hearts were 147, 154 and 127 beats/min, which were obtained from their subject-specific ultrasound images, and which fitted within variability range in the literature. $^{47}$

\section{Disease Features}

Upon calibration of the healthy heart models, we adjusted parameters of to model the following disease features:

1. Aortic stenosis. To simulate this, we increased aortic flow resistance in the Windkessel model, and considered 3 levels of severity, a 3-fold, 10fold, and 100-fold increase in aortic resistance, and denote these scenarios as " $\mathrm{R}_{\mathrm{AO}} * 3$ ", " $\mathrm{R}_{\mathrm{AO}} * 10$ ", and " $\mathrm{R}_{\mathrm{AO}} * 100$ ", respectively

2. Aortic stenosis and mitral regurgitation. MR often accompanies fetal AS. ${ }^{11}$ To model this, additional Windkessel elements were added to the AS cases to allow for systolic MR flow (Fig. 2). Three severity levels were again modelled, with the MR flow resistance being $10^{6}, 10^{4}$, and $10^{3}$ times that of the forward mitral inflow resistance as shown in Fig. 2 dotted line. We denote these scenarios as " $\mathrm{R}_{\mathrm{MR}} 10^{6 ",}, " \mathrm{R}_{\mathrm{MR}} 10^{4}$ ", and " $\mathrm{R}_{\mathrm{MR}} 10^{3 "}$,, respectively. The case of " $\mathrm{R}_{\mathrm{AO}} * 100, \mathrm{R}_{\mathrm{MR}} 10^{3}$ " demonstrated valve velocities results that were close to clinical observations in our critical AS cases. This model was used as the baseline for modelling disease features $3-5$ below

3. LV hypertrophy. Increased wall thickness can accompany critical $\mathrm{AS}^{31}$ To model this, the epicardial boundary of our LV geometry was offset outwards to generate thicker LV walls without reducing luminal volume. We investigated $10 \%, 25 \%, 50 \%$ and $100 \%$ increase in wall thickness

4. Endocardial Fibroelastosis. Endocardial fibroelastosis is commonly observed for fetal hearts with critical $\mathrm{AS}^{32}$ We thus investigate the effects of myocardial stiffness changes, using scenarios of stiffness increased by $50 \%$ and $100 \%$, via scaling of parameter C in Eq. (1)

5. Cardiomyopathy. Cardiac contractility can be weakened in some cases of $\mathrm{AS}^{.24} \mathrm{We}$ investigated scenarios where the active tension was reduced, by reducing $\mathrm{T}_{\max }$ in Eq. (3) to $66 \%$ and $50 \%$ of its original value 
Valve Flow Velocity Formulation

From the FE and Windkessel model, flow at the valves was modelled as volume flow rates. However, valve flow is typically quantified as Doppler velocities in clinical measurements, and volume flow rate is difficult to measure directly, due to difficulties in accurately measuring valve orifice size and acquiring good Proximal Iso-velocity Surface Area (PISA) images. We thus adopted an empirical formulation to convert volume flow rate to flow velocities to enable clinical interpretations of our results. Valve volume flow rate data was converted to flow velocities via the discharge coefficient $\left(C_{d}\right)$ formulation. We adopted the empirical $C_{d}$ formula below, ${ }^{12}$ which was based on work by Nakayama et al. ${ }^{38}$ via studies of jet flow through small orifices:

$$
C_{d}=\frac{\mathrm{Re}^{\frac{5}{6}}}{17.11 \frac{l}{d}+1.65 \mathrm{Re}^{0.8}}
$$

where $l$ and $d$ are the axial length and the radial diameter of the orifice respectively, and $R e$ is the Reynolds number, calculated with the average velocity within the orifice, diameter of the orifice, and fluid viscosity. We adopted a kinematic viscosity of $4 * 10^{-6}$ $\left(\mathrm{m}^{2} / \mathrm{s}\right)$ from the literature. ${ }^{4}$ Nakayama et al.'s model was considered because it was formulated for $l / d$ in the range of 1.5 to 17 and Re in the range 550 to 7000 , and the conditions found in our study were within these ranges.

The thickness of the heart valves can be used to estimate the axial length of the orifice flow. We estimated the thickness of the aortic and mitral valves of fetal heart by first obtaining literature value of newborn heart valve leaflet thicknesses ${ }^{44}$ and then scaling them down assuming a direct linear relationship with cardiac diameter. ${ }^{19}$ Thickness values used were 0.25 and $0.27 \mathrm{~mm}$ for the mitral and aortic valves. These thickness values were then assumed to be the axial length of the stenotic / regurgitant valve orifices.

$C_{d}$ can be equated to pressures and flow rates via the Euler number $(E u)$,

$$
E u=\frac{1}{C_{d}^{2}}=\frac{\Delta P}{\frac{1}{2} \rho u^{2}}
$$

where $\Delta \mathrm{P}$ is pressure difference across the orifice, $\rho$ is the blood density $\left(1000 \mathrm{~kg} / \mathrm{m}^{3}\right),{ }^{36} u$ is average velocity within the orifice, which can be calculated from the volume flow rate and orifice size. Equation (5) was then solved with a simple gradient descent algorithm as follows (Eq. 6), and its results were manually verified.

$$
\arg \min _{d}\left(\frac{1}{2} \rho u^{2}-\Delta P C_{d}^{2}\right)
$$

\section{RESULTS}

The end-systolic myocardium geometry produced by FE modelling matched well with that produced by cardiac motion estimation from ultrasound images (Fig. 3). The average surface distance error was quantified to be less than $0.060 \mathrm{~mm}$ for all 3 hearts, which was less than $1 \%$ of the diameter of the smallest heart. The end-diastolic geometry from both analyses also matched well, but this was a trivial result since the end-diastolic geometry was the starting geometry for both analyses. A further quantification at 5 different time points spread out over the cardiac cycle (demonstrated in supplementary Fig. S4) showed that the distance error was were $0.038 \pm 0.018 \mathrm{~mm}$, $0.058 \pm 0.033 \mathrm{~mm}$, and $0.070 \pm 0.030 \mathrm{~mm}$ of the respective for the 22, 28 and 32 weeks old hearts.

In addition, the LV volumes and stroke volume predicted by the models matched those obtained from ultrasound images (Table 1). Further, the stroke volume and chamber volumes of the 22 and 28 weeks old fetal hearts in this study were close to the mean values of their age-matched population reported in the literature, suggesting that they are suitable representation of their population. The 32 weeks old cases, however, was larger than the average of its age-matched population.

\section{Effects of Aortic Stenosis and Mitral Regurgitation}

Systolic pressure increased and stroke volume decreased with increasing AS severity, which was simulated by increasing the aortic valve resistance (Fig. 4a). The results showed that the $\mathrm{R}_{\mathrm{AO}} * 100$ was a sufficiently severe condition with stroke volume reducing to near zero. At this condition, LV pressures became elevated by about $10-20 \mathrm{mmHg}$ above their healthy state. When MR was included to the AS cases, stroke volumes were partially restored and peak pressures were decreased (Fig. 4b). Generally, pressure reduction was modest (up to about $5 \mathrm{mmHg}$ ) for the range of mitral flow resistances explored.

Figures $5 \mathrm{a}$ and $5 \mathrm{~b}$ supplementary video 2 shows the spatial patterns of the fiber strain for the 32 weeks old fetal heart model in healthy and diseased $\left(\mathrm{R}_{\mathrm{AO}} * 100\right.$ $\mathrm{R}_{\mathrm{MR}} 10^{3}$ ) conditions. The spatial strain pattern was largely similar between the healthy and diseased cases, but the magnitude was different and reduced with AS. Figure $5 \mathrm{~b}$, which plots the temporal waveform of the spatially averaged longitudinal and circumferential strains for the 32 weeks old fetal heart model, demonstrates this further. With AS alone, both longitudinal and circumferential strains magnitude reduced with increasing severity of the AS. The decrease in circumferential strain magnitude was greater than that 


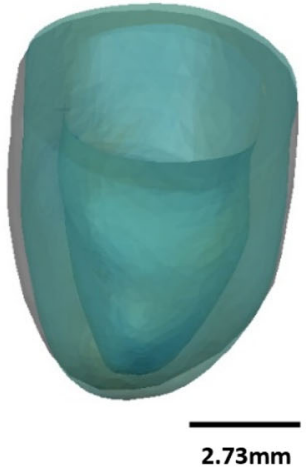

22 weeks

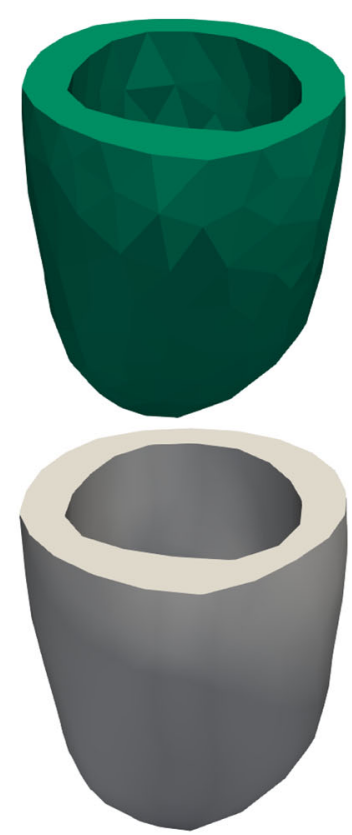

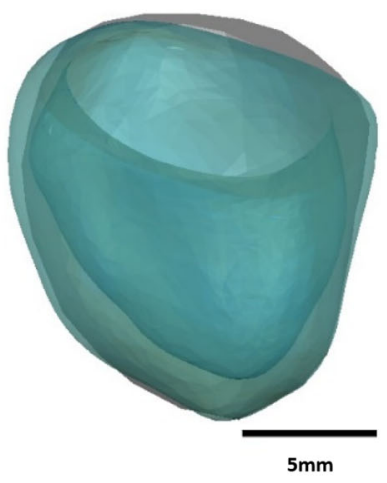

28 weeks
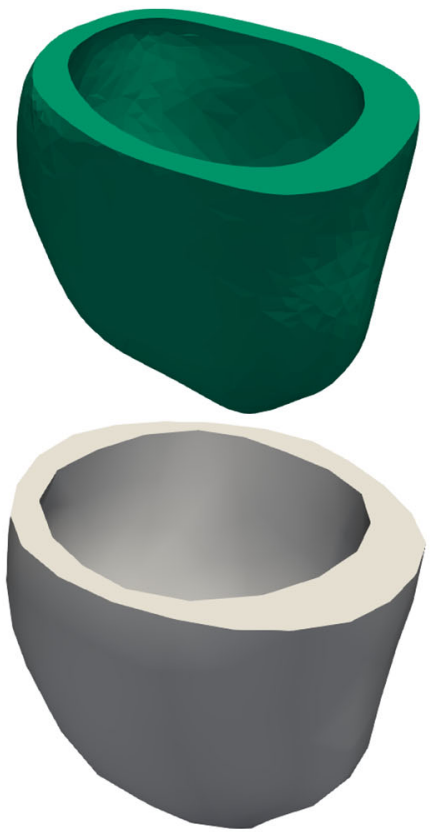

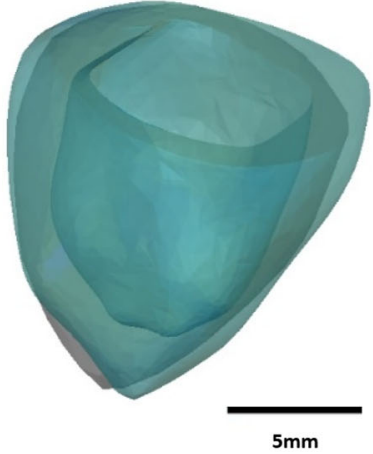

32 weeks

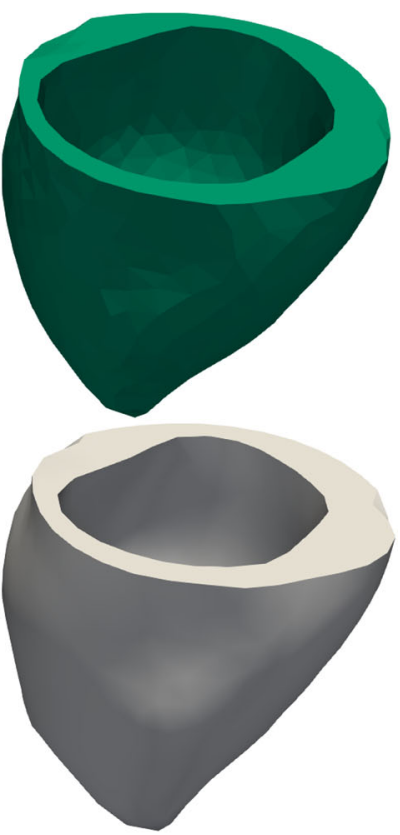

FIGURE 3. The end-systolic LV geometries of the healthy fetal hearts studied, obtained from FE modelling (green), and from ultrasound image motion tracking (gray). Geometries from both analyses showed a good match. The input to both FE modelling and motion tracking were the segmentation of the end-diastolic LV geometry.

in longitudinal strain magnitude. Strain could be reduced to near zero value at $\mathrm{R}_{\mathrm{AO}} * 100$. With $\mathrm{MR}$, this decrease in strain were less pronounced, which was similar to observations for stroke volume. Peak strains for all hearts are summarized in supplementary Fig. S1, and reflect largely the same trend as in Fig. 5.

Stress in the myofiber direction generally increased with gestational age. From 22 to 32 weeks, this stress increased by $43.1 \%$ (Fig. 6a). This increase can be attributed to a $24 \%$ increase in systolic pressures, an $83 \%$ increase in LV luminal space, and a $\sim 56.4 \%$ increase in wall thickness in the same period. Stress in the myofiber direction also increased with AS, with the increase approximately proportional with the elevations of LV systolic pressures (Fig. 6a). With MR, the stress decreased in the stenotic hearts (Figs. 6b and 6c). Figure $6 \mathrm{~b}$ shows that the spatially-averaged stress waveform was similar to the LV pressure waveform. Similar data for the 22 and 28 weeks old fetal hearts are given in Figs. S2 and S3 in the supplementary text.

Interestingly, with AS and MR, the systole-diastole timing was changed, as could be observed from the flow rate waveforms across the valve (Figs. 7a and 7b). A decrease in the mitral inflow diastolic duration and an increase in the aortic outflow duration was observed. With AS alone, diastolic duration could be reduced by up to $74 \%$ in the most severe simulation for 28 weeks heart, up to $68 \%$ for the 28 weeks heart, and up to $88 \%$ for the 32 weeks heart. When MR accompanied AS, this reduction was partially alleviated and diastolic duration was increased slightly. The same observation can be made from Doppler data of our KUH dataset, where systole-diastole duration ratios was found to be $3.16 \pm 1.57$, which meant $74 \pm 10 \%$ 
TABLE 1. Volumes and pressures of the FE LV normal fetal hearts studied, compared to quantifications from their 4D ultrasound images, and compared to literature values of their age-matching cohort

\begin{tabular}{|c|c|c|c|}
\hline & Literature $(n=63)$ & Clinical Image & FE Model \\
\hline \multicolumn{4}{|l|}{22 weeks } \\
\hline End diastole volume (ml) & $1.17 \pm 0.28$ & 0.94 & 0.89 \\
\hline End systole volume (ml) & $0.60 \pm 0.19$ & 0.60 & 0.55 \\
\hline Stroke volume (ml) & $0.54 \pm 0.16$ & 0.35 & 0.36 \\
\hline Pressure (mmHg) & 27.0 & & 30.12 \\
\hline \multicolumn{4}{|l|}{28 weeks } \\
\hline End diastole volume (ml) & $2.89 \pm 0.68$ & 2.05 & 2.15 \\
\hline End systole volume (ml) & $1.61 \pm 0.45$ & 1.17 & 1.32 \\
\hline Stroke volume (ml) & $1.30 \pm 0.36$ & 0.88 & 0.83 \\
\hline Pressure (mmHg) & 37.2 & & 32.87 \\
\hline \multicolumn{4}{|l|}{32 weeks } \\
\hline End diastole volume (ml) & $3.27 \pm 0.86$ & 5.22 & 5.45 \\
\hline End systole volume (ml) & $1.95 \pm 0.56$ & 3.72 & 3.87 \\
\hline Stroke volume (ml) & $1.41 \pm 0.44$ & 1.49 & 1.59 \\
\hline Pressure (mmHg) & 44.2 & & 40.2 \\
\hline
\end{tabular}

End-diastole and end-systole volumes and stroke volumes (means \pm SD) were obtained from Ref. 48, while pressures were calculated with an empirical equation from Ref. 25.

of the cardiac cycle was systole, and $26 \pm 10 \%$ was diastole. A sample Doppler image is shown in Fig. 7c.

The valve velocity estimated using the coefficient of discharge formulation is shown in Table 2 for the 28 weeks old fetal heart (also plotted in supplementary Fig. S5). Data for the other 2 hearts shows largely the same trends and are given in the supplementary Tables S4 and S5. Aortic outflow and MR velocities of the cohort of 32 critical AS fetal cases are also given for comparison. The aortic valve velocities from stenotic heart simulations were higher than literature values of aortic valve velocities for healthy hearts. Interestingly, we found that even when the AS was sufficiently severe to reduce stroke volume to close to zero, the aortic velocity could remain high, above $2 \mathrm{~m} /$ s. More severe AS was found necessary to decrease the aortic velocity further. This suggested that fetal LV stroke volume might be more sensitive to AS severity than aortic velocity. Our data also showed that at a milder stenosis, an increase in stenosis level (from $\mathrm{R}_{\mathrm{AO}} * 3$ to $\mathrm{R}_{\mathrm{AO}} * 10$ ) led to an increase in aortic velocity, but at a more severe stenosis, increasing stenosis (from $\mathrm{R}_{\mathrm{AO}} * 10$ to $\left.\mathrm{R}_{\mathrm{AO}} * 100\right)$ led to a decrease in aortic velocity.

We further found that the aortic velocities in our simulated stenotic cases were close to the mean of the peak aortic Doppler velocities measured for our diseased fetal heart cohort $(2.35 \mathrm{~m} / \mathrm{s})$. However, for peak MR velocities, our simulation results showed a range from close to $0 \mathrm{~m} / \mathrm{s}$ to less than $3 \mathrm{~m} / \mathrm{s}$, which were lower than that clinically measured in our diseased cohort $(3.40 \mathrm{~m} / \mathrm{s})$. Further manipulation of the MR resistance could not increase mitral velocities above $3 \mathrm{~m} / \mathrm{s}$, unless thickening of the LV wall was modelled, as described in analysis below. We thus noted that LV remodelling, such as hypertrophy, appeared to be necessary to increase MR velocities above $3 \mathrm{~m} / \mathrm{s}$.

\section{Effects of LV Hypertrophy}

Manual quantification of the LV free wall thickness ( 3 measurements per echo image, 3 echo images per case) was conducted in 10 randomly selected cases from our KUH data set (average age $=28.0 \pm 1.6$ weeks). The free wall thickness was found to be $6.0 \pm 1.1 \mathrm{~mm}$, which is $105 \pm 37 \%$ larger compared to value reported ${ }^{29}$ for age-matched healthy fetal hearts $(2.9 \pm 0.3 \mathrm{~mm})$. The wall thicknesses of the 3 healthy fetal heart models used in our study were: 2.1 , 2.9 and $3.3 \mathrm{~mm}$.

The results of simulations with increased $\mathrm{LV}$ wall thicknesses for the case of $\mathrm{R}_{\mathrm{AO}} * 100, \mathrm{R}_{\mathrm{MR}} 10^{3}$ are shown in Figs. $8 \mathrm{a}-8 \mathrm{c}$ and Table $3 \mathrm{~A}$. With increasing wall thickness, stroke volume and LV systolic pressure increased, but myocardial stress in the fiber direction decreased. A $50 \%$ increase in thickness increased the peak systolic pressure by $72 \%$, increased valve flow velocities from $2.8 \mathrm{~m} / \mathrm{s}$ to $3.9 \mathrm{~m} / \mathrm{s}$, increased stroke volume by $54 \%$, increased LV wall strain in the fiber direction by $35 \%$, but decreased myocardial stress in the fiber direction by $25 \%$. In the $100 \%$ hypertrophy simulation case, however, the LV systolic pressure was $97.5 \mathrm{mmHg}$, higher than average value of approximately $63 \mathrm{mmHg}$ reported for fetal AS cases. ${ }^{53}$ The valve velocities (Table 3A) were much higher than the average in our KUH dataset (Table 2), even though $100 \%$ hypertrophy was found to be the mean level of hypertrophy in our AS cases. This suggested that re- 
(a)

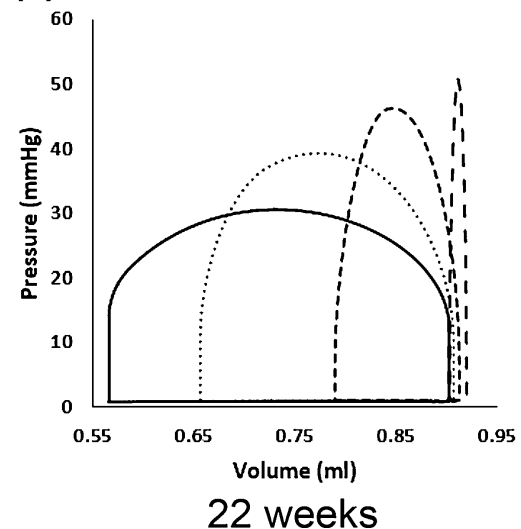

Healthy

(b)
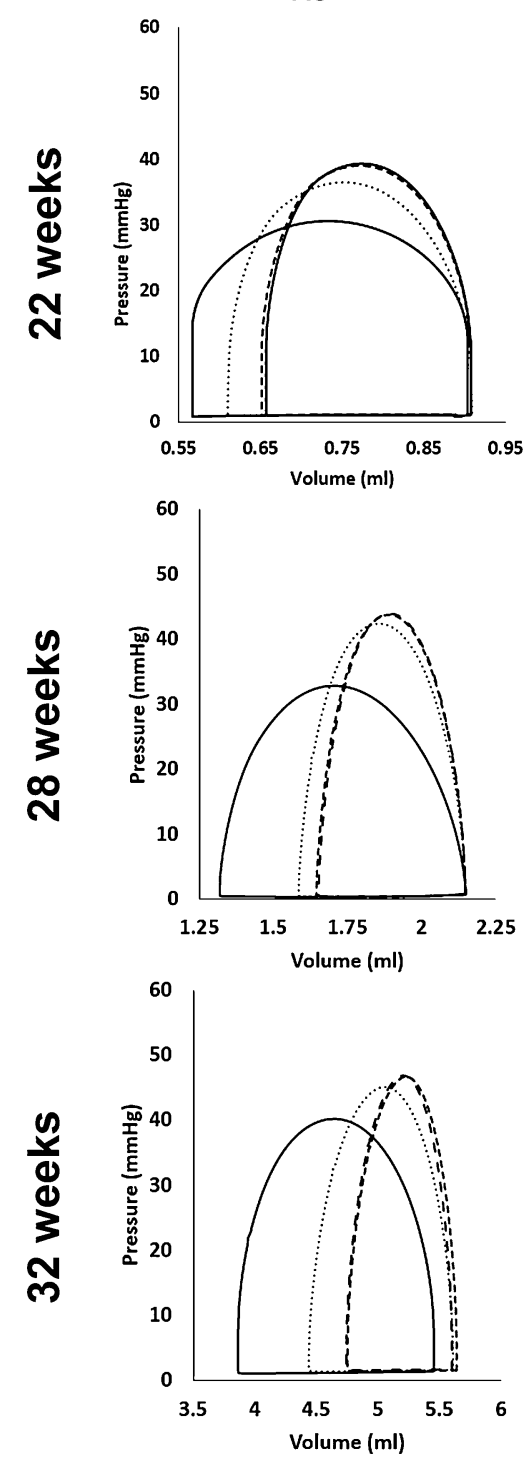

-Healthy

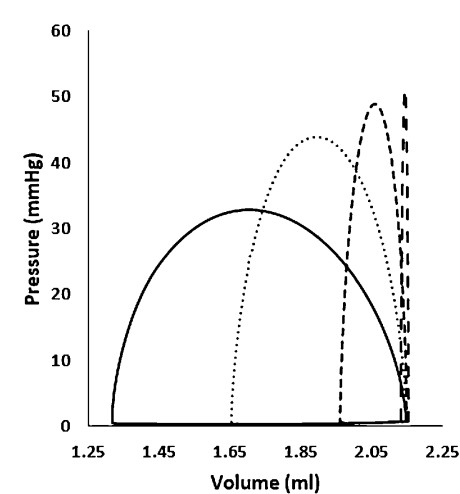

28 weeks

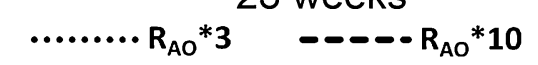

$R_{\mathrm{AO}} * 10$
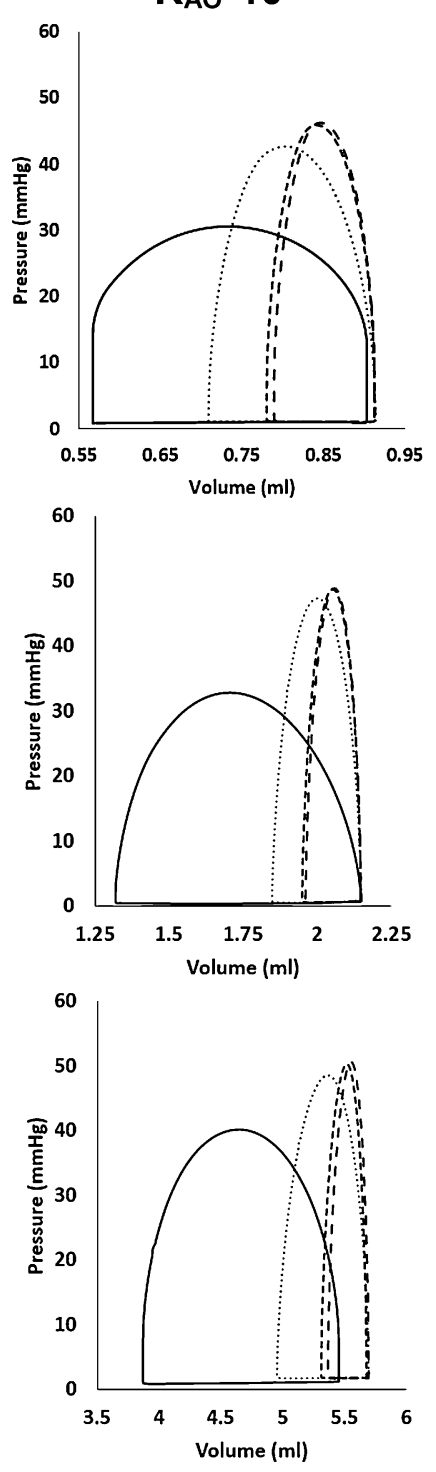

$\mathrm{R}_{\mathrm{MR}} \mathbf{1 0}^{\mathbf{3}}$

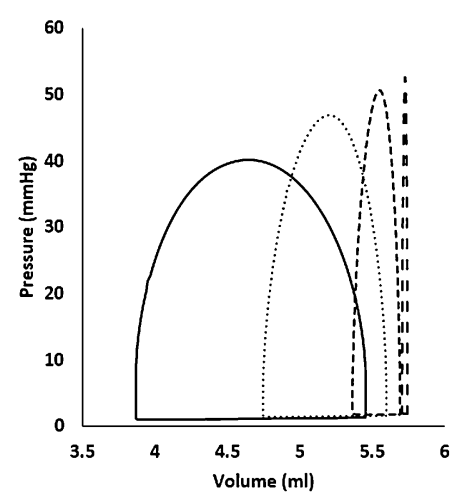

32 weeks
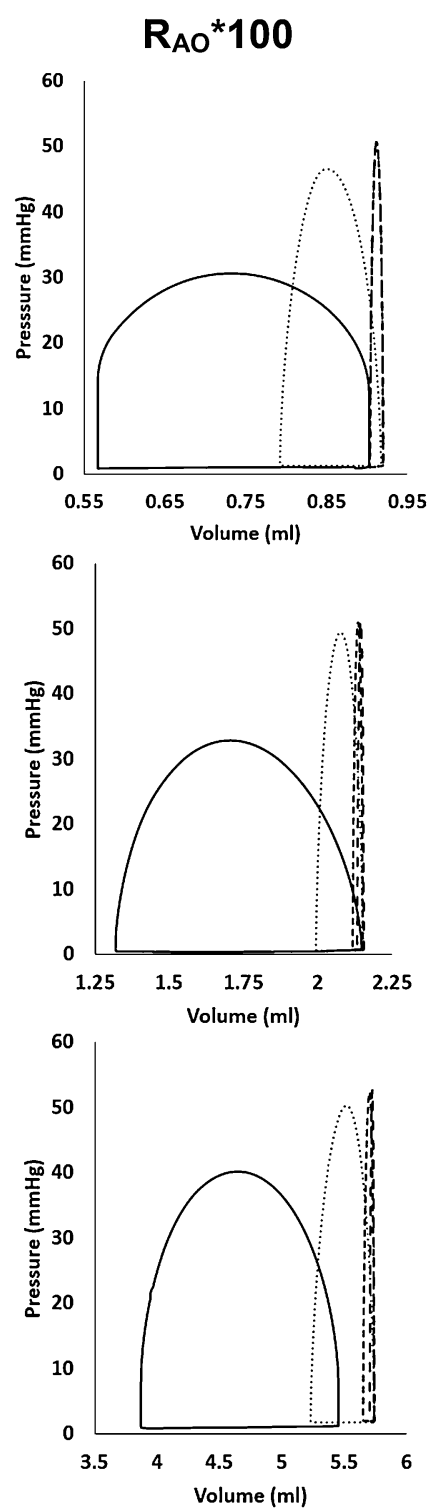

$---\mathbf{R}_{\mathrm{MR}} 10^{6}$ 
4FIGURE 4. PV loop from FE simulations of healthy fetal hearts of various gestational ages, and the same hearts with various disease severity. (a) AS alone compared to healthy hearts. (b) AS with MR compared to healthy hearts. duced contractility is likely to have accompanied AS cases with hypertrophy.

It was noteworthy that, after simulations of multiple cardiac cycles, thicker wall simulation cases demon- (a)

Mid

Systole
Healthy 32 weeks old case

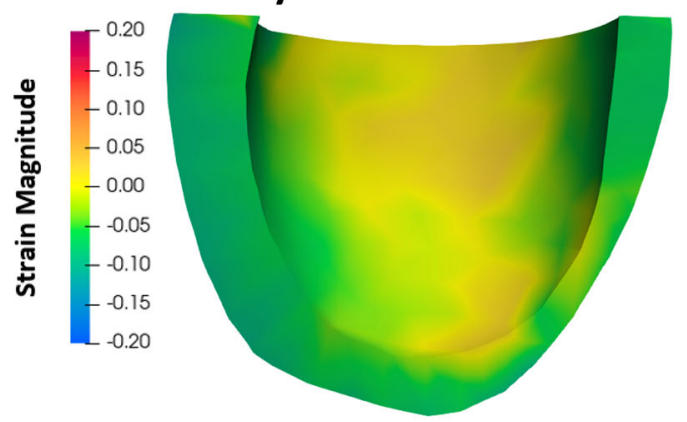

End

Systole
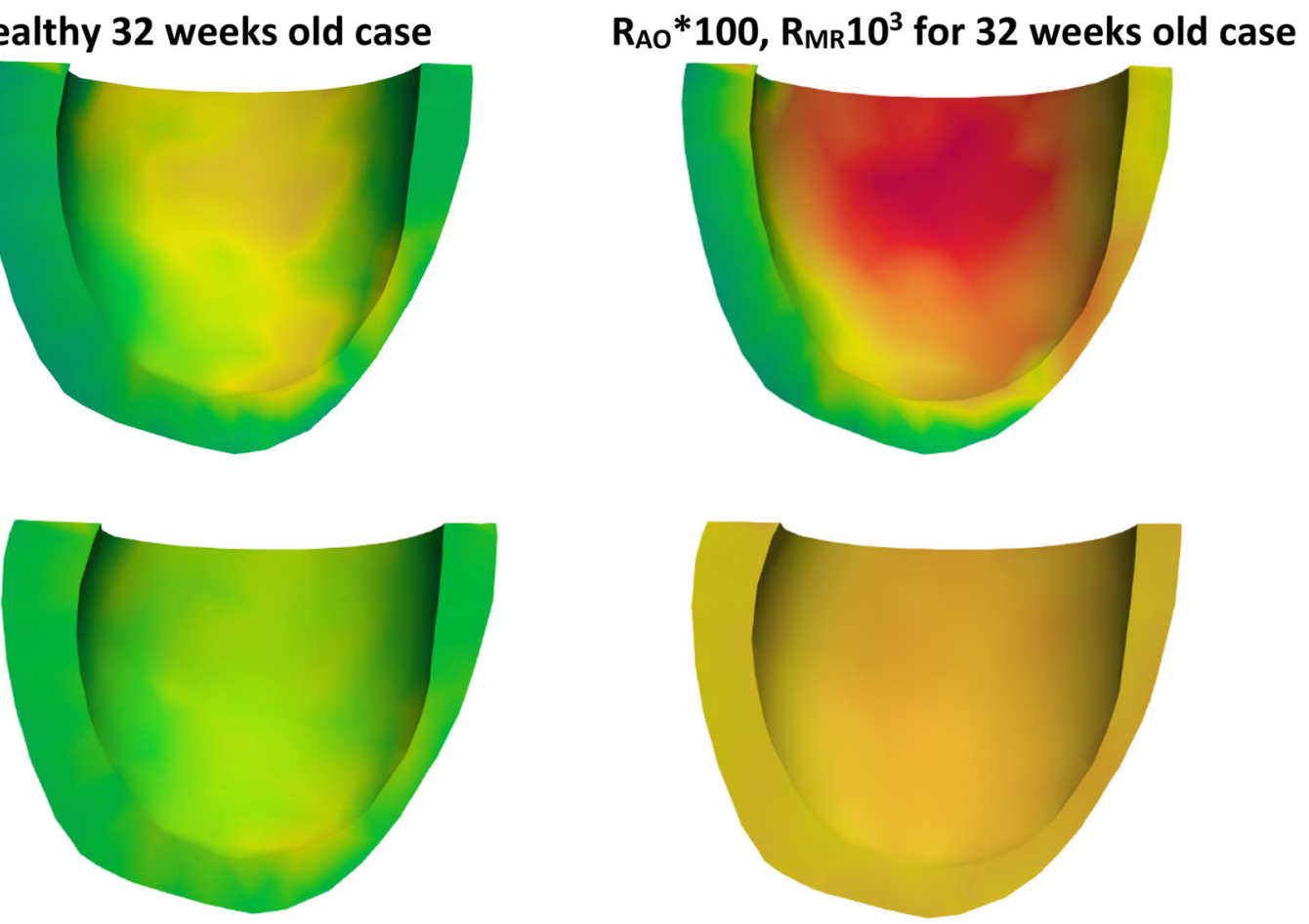

(b)

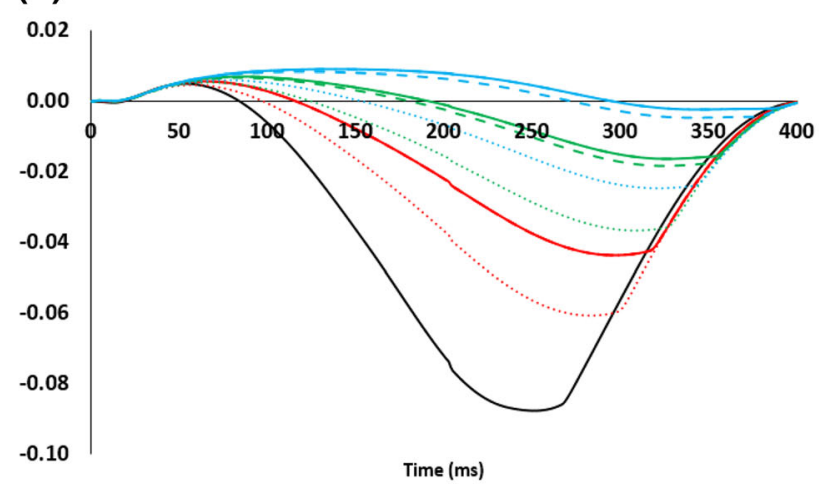

(i) Circumferential Strain

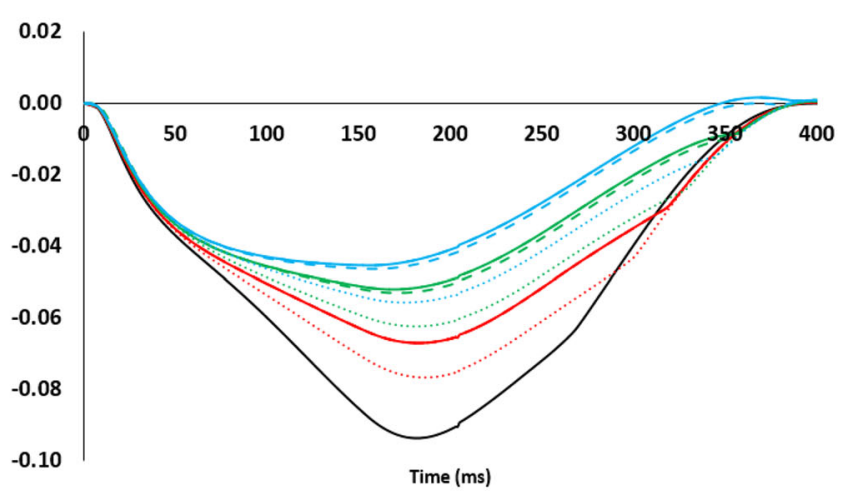

(ii) Longitudinal Strain

$$
\begin{aligned}
& \text { - Healthy } \\
& -\mathrm{R}_{\mathrm{AO}} * 3 \quad \cdots \cdots \cdot \mathrm{R}_{\mathrm{AO}} * 3 \mathrm{R}_{\mathrm{MR}} 10^{3} \quad--\mathrm{R}_{\mathrm{AO}} * 3 \mathrm{R}_{\mathrm{MR}} 10^{4} \quad \cdots-\mathbf{R}_{\mathrm{AO}} * 3 \mathbf{R}_{\mathrm{MR}} 10^{6} \\
& -\mathrm{R}_{\mathrm{AO}} * 10 \quad \cdots \cdots . . . \mathrm{R}_{\mathrm{AO}} * 10 \mathrm{R}_{\mathrm{MR}} 10^{3}---\mathrm{R}_{\mathrm{AO}} * 10 \mathrm{R}_{\mathrm{MR}} 10^{4}-\cdot-\mathrm{R}_{\mathrm{AO}} * 10 \mathrm{R}_{\mathrm{MR}} 10^{6} \\
& \mathrm{R}_{\mathrm{AO}} * 100 \quad \cdots \cdots \cdot \mathrm{R}_{\mathrm{AO}} * 100 \mathrm{R}_{\mathrm{MR}} 10^{3}---\mathrm{R}_{\mathrm{AO}} * 100 \mathrm{R}_{\mathrm{MR}} 10^{4}-\cdot-\mathrm{R}_{\mathrm{AO}} * 100 \mathrm{R}_{\mathrm{MR}} 10^{6}
\end{aligned}
$$

FIGURE 5. (a) Spatial pattern of fiber-direction strain for healthy and diseased $\left(R_{A O}{ }^{*} 100, R_{M R} 10^{3}\right)$ case for 32 weeks old fetal heart. (b) Spatially-averaged circumferential and longitudital strains over time of the 32 weeks old fetal heart, for the healthy and diseased (various severity) cases. 

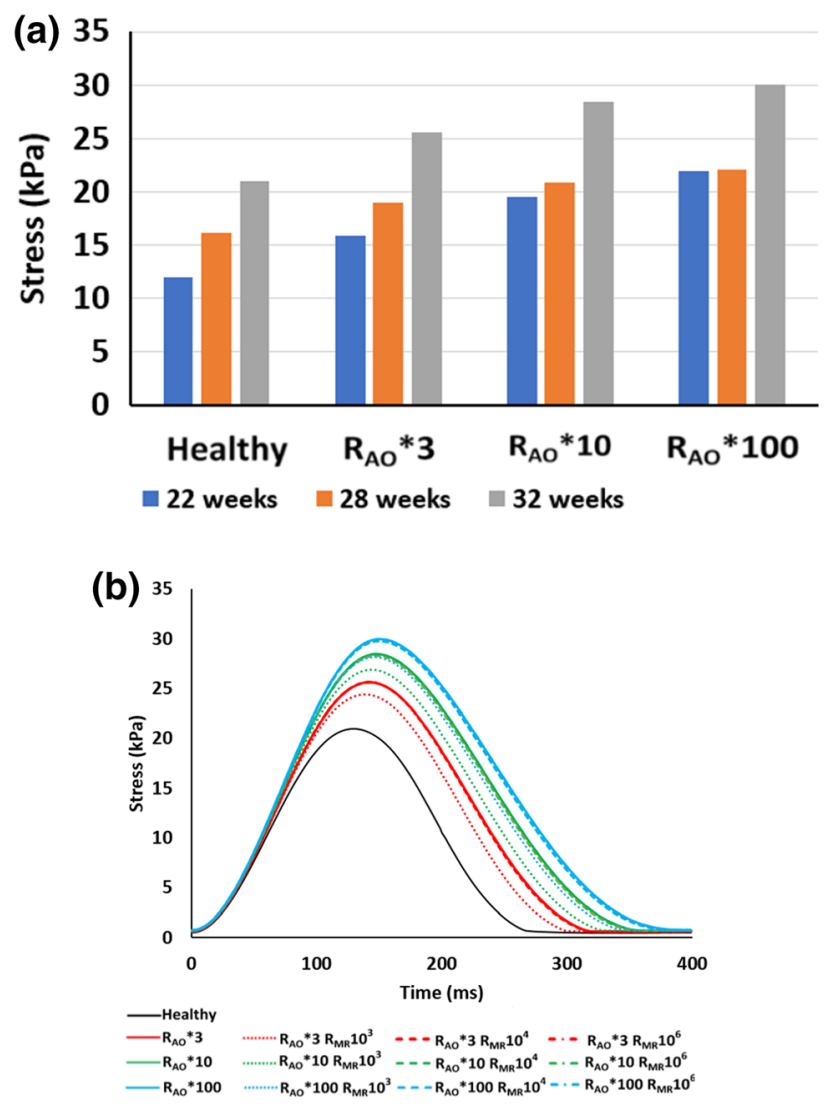

(c)

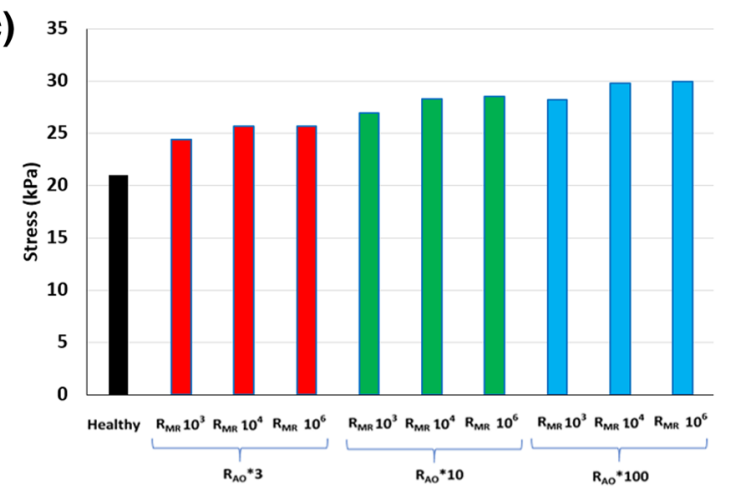

FIGURE 6. (a) Temporal peak of the spatial-averaged stress in the myofiber direction, for hearts of various gestational age, in health and during AS. (b) Spatially-averaged stress in the myofiber direction across time for the 32 weeks old fetal heart, comparing healthy and various disease scenarios. (c) Quantification of the temporal peak stresses from (b).

strated a reduction of the overall LV lumen size compared to the baseline case before thickening. This occurs even though we have modelled thickening only in the epicardial direction as the initial condition. This is because thicker walls reduced LV compliance, ${ }^{35}$ which slightly inhibited diastolic distention.

\section{Effects of Fibroelastosis}

We used stiffness changes as a model for fibroelastosis. Results are shown in Figs. 8d-8f and Table 3B. Surprisingly, a substantial $50 \%$ increase in myocardial tissue stiffness produces only modest reduction of the peak systolic pressure $(4.4 \%)$, ventricular size $(6.1 \%)$, stroke volume $(6.8 \%)$, and stress in myofiber direction $(5.3 \%)$. Table 3B shows that aortic outflow and MR velocities were only mildly reduced.

\section{Effects of Cardiomyopathy}

Results of modelling with decreased LV contractility are shown in Figs. $8 \mathrm{~g}-8 \mathrm{i}$ and Table 3C. Unsurprisingly, the reduction produced a roughly proportional decrease in stroke volume and systolic pressure. Corresponding decrease in stress (myofiber direction), strains, reduced aortic outflow and MR velocities were also observed.

\section{DISCUSSION}

In this study, we developed and calibrated FE models of healthy fetal hearts from 4D ultrasound images and Doppler velocity measurements, and then used them to investigate biomechanical changes during disease conditions associated with fetal AS. We could successfully calibrate the FE models, where healthy heart models showed stroke volume, chamber sizes, and LV geometry that agreed well with the same data obtained from clinical images. When we used the calibrated FE model to investigate diseased scenarios, we could capture systole-diastole timing alterations observed in clinical measurements in disease cases, and our simulated valve velocity results were close to these measurements. The observation of alteration of systole-diastole timing under critical aortic stenosis cases was also reported in the literature. ${ }^{8}$ These good agreements between the simulation results and clinical measurements suggested that the FE models may be able to predict fetal heart biomechanics well, and may be a useful tool to understand normal and diseased heart physiology. FE modelling is further useful as it can estimate parameters that are difficult to measure in vivo, such as myocardial stresses.

The focus of our current work, however, was to investigate the individual effects of each of the disease features. For this reason, we did not study the interdependencies of the various features, and we used the healthy heart as a baseline for our investigation, instead of directly using the diseased heart anatomy and diseased myocardial characteristics as the baseline. This is also due to the lack of information of the dis- 
(a)
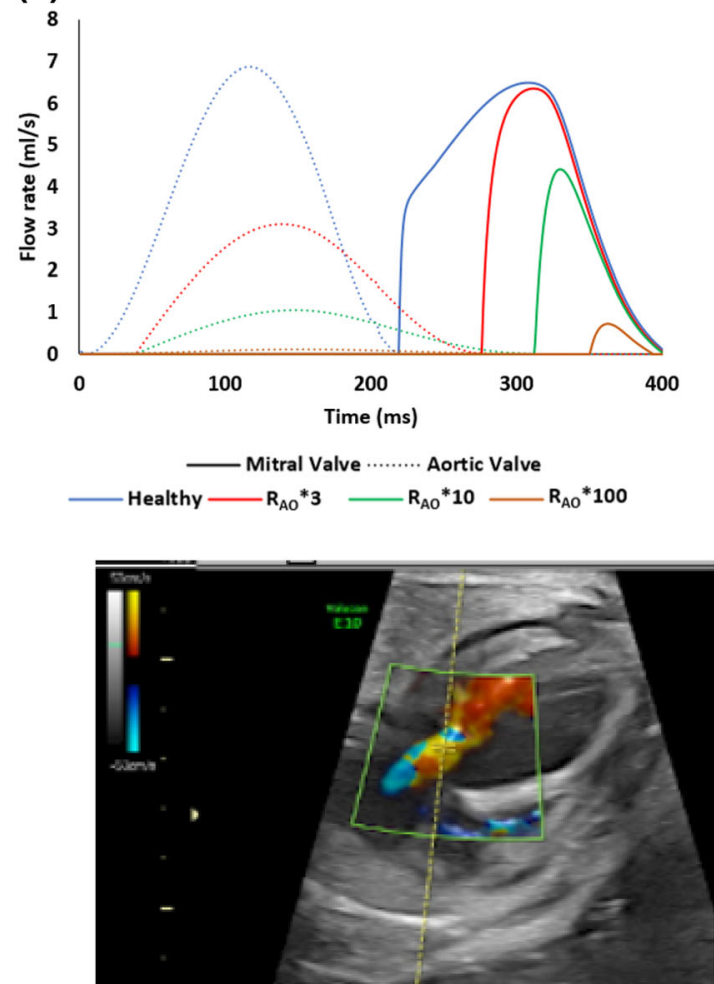

(b)
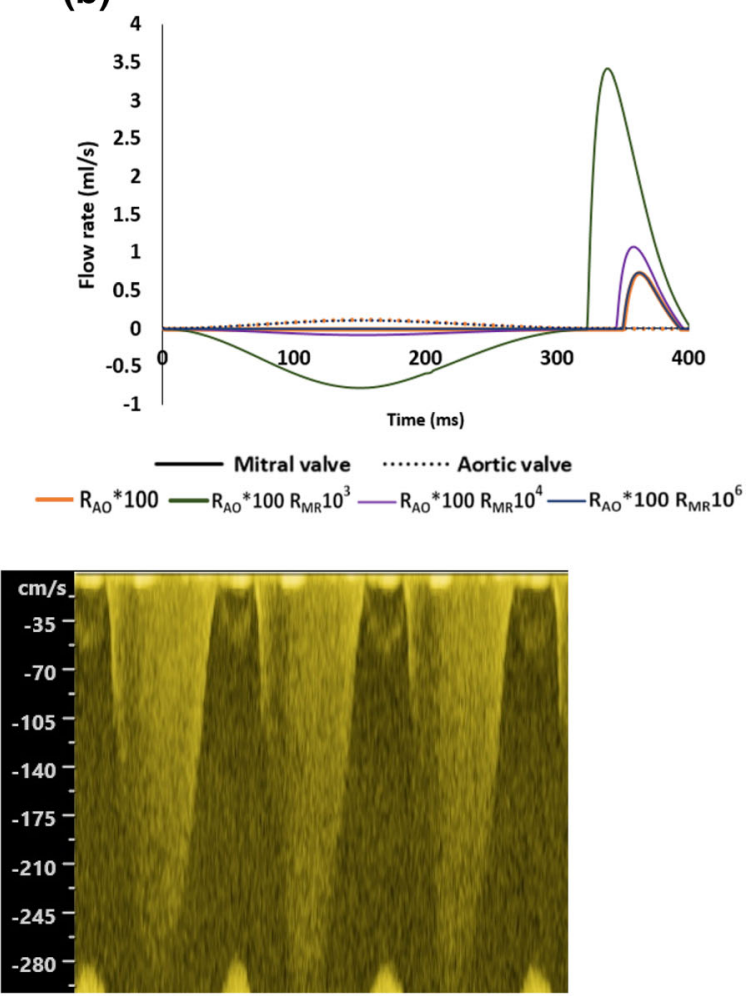

FIGURE 7. (a, b) Flow rates at the mitral and aortic valves under healthy and different disease condition for the 28 weeks old fetal heart. (a) Healthy case compared to cases with only AS. (b) The AS case of $R_{A O} * 100$ compared to the same case with various severity of MR. (c) Mitral Doppler velocity measurements in a 29 weeks old fetal heart with AS and MR, demonstrating long durations of regurgitation but short durations of mitral inflow.

TABLE 2. Peak flow velocities $(\mathrm{m} / \mathrm{s})$ at the valves of fetal hearts at various gestational ages, calculated based on discharge coefficient formulation for various diseased scenarios, compared to normal hearts from the literature; ${ }^{2}$ and the average velocities measured via Doppler ultrasound from our cohort of clinical AS and MR cases.

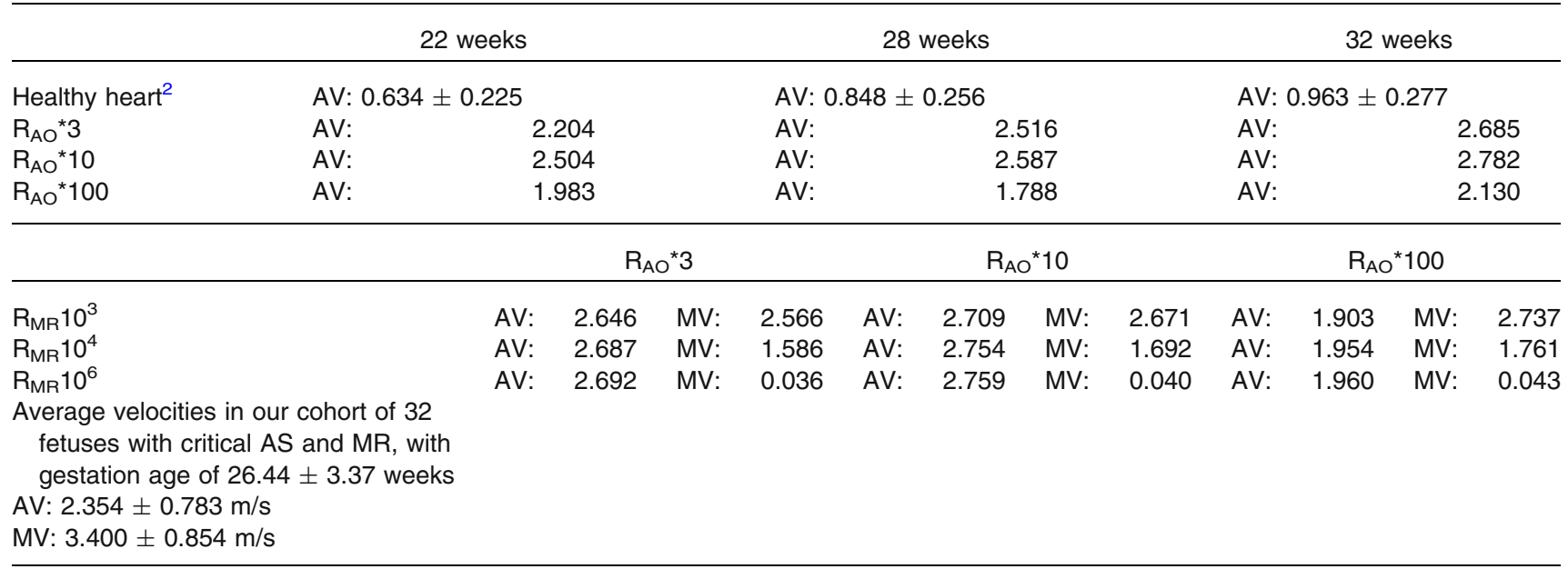

AV: aortic valve, MV: mitral valve.

eased fetal myocardium. These are limitations of the current work that warrants future studies. However, the advantage of our chosen focus is that we can better understand the effects of each individual disease feature, which will be difficult with clinical or animal studies. Simulations results from the healthy baseline 
(a)

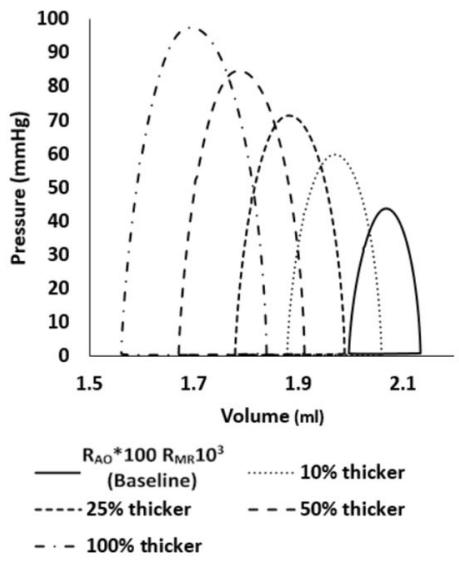

(d)

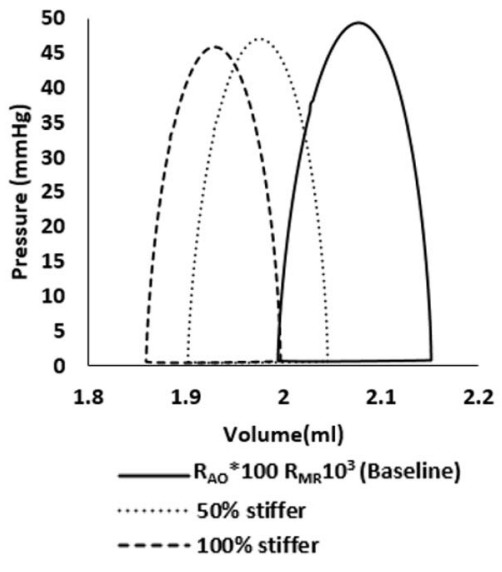

(h)

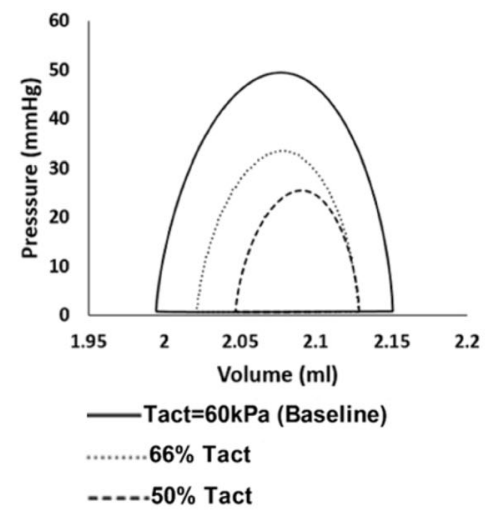

(b)

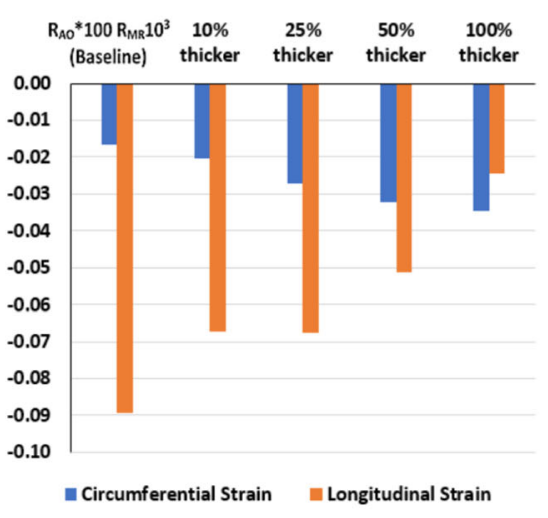

(e)

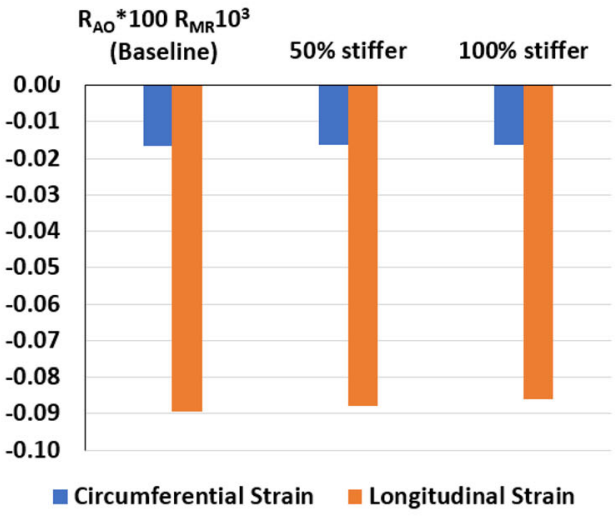

(i)

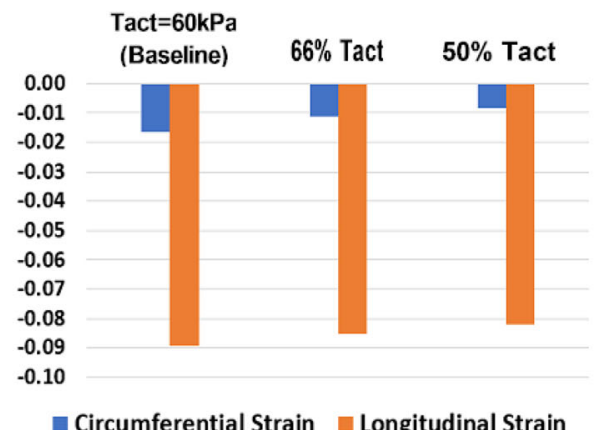

घ Circumferential Strain $\quad$ Longitudinal Strain (c)

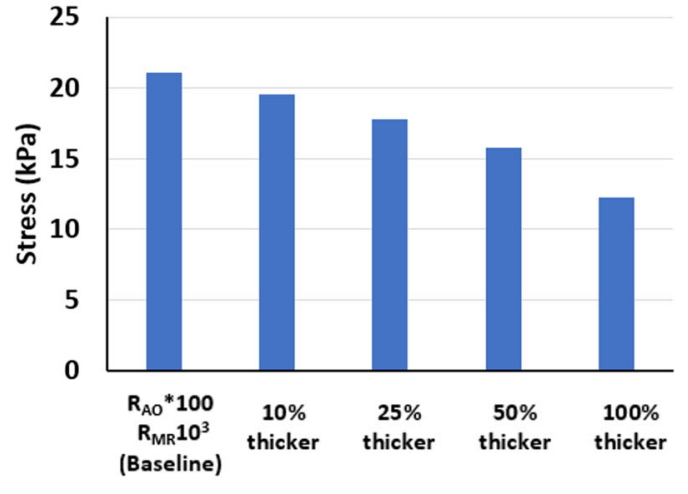

(f)

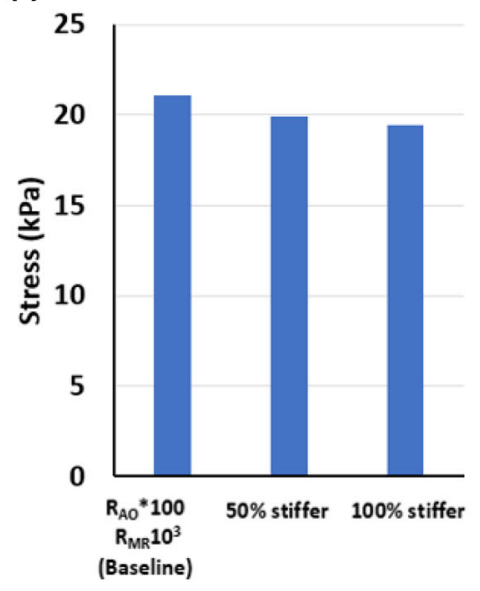

(g)

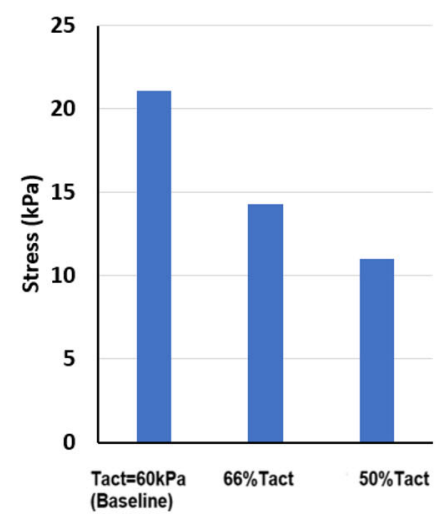

FIGURE 8. Effect of (a-c) LV wall thickening, (d-f) increasing LV stiffness, and ( $g-i)$ decreasing LV contractility on the (a, $d, g)$ PV loop, (b, e, h) temporal-peak, spatial-averaged circumferential strain and longitudinal strain, and (c, f, i) temporal-peak, spatialaveraged myocardial stress in the fiber direction.

can also be helpful when considering the next question of how abnormal biomechanics can lead to pathological remodelling.
From our results, we found that AS can elevate fetal LV peak systolic pressures significantly (10$20 \mathrm{mmHg}$ ), but MR can reduce this slightly. With 
TABLE 3. Peak flow velocities $(\mathrm{m} / \mathrm{s})$ for $(A)$ various wall thickness cases, (B) various wall stiffness cases, and (C) various LV contractility cases, modelled from the $R_{A O} * 100$, $\mathrm{R}_{\mathrm{MR}} 10^{3}$ baseline condition

Case $\quad$ Velocity $(\mathrm{m} / \mathrm{s})$

(A) Effects of Increasing $L V$ wall thickness

$\mathrm{R}_{\mathrm{AO}}{ }^{*} 100, \mathrm{R}_{\mathrm{MR}} 10^{3}$ (baseline) $\quad \mathrm{AV}: \quad 1.903 \quad \mathrm{MV}: \quad 2.737$

$10 \%$ wall thickness increase $\quad$ AV: 2.262 MV: 3.076

$25 \%$ wall thickness increase $\quad$ AV: 2.680 MV: 3.465

$50 \%$ wall thickness increase $\quad$ AV: 3.179 MV: 3.881

$100 \%$ wall thickness increase $\quad$ AV: $\quad 3.544$ MV: 4.260

(B) Effects of Increasing $L V$ wall stiffness

$\mathrm{R}_{\mathrm{AO}}{ }^{*} 100, \mathrm{R}_{\mathrm{MR}} 10^{3}$ (baseline) $\quad \mathrm{AV}: \quad 1.903 \mathrm{MV}: \quad 2.737$

$50 \%$ stiffness increase $\quad$ AV: 1.872 MV: 2.707

$100 \%$ stiffness increase $\quad$ AV: 1.884 MV: 2.718

(C) Effects of decreasing LV contractility

$\mathrm{T}_{\max }=60 \mathrm{kPa} \quad \mathrm{AV}: \quad 1.903 \quad \mathrm{MV}: \quad 2.737$

$33 \%$ decrease in $\mathrm{T}_{\max }(40 \mathrm{kPa}) \quad \mathrm{AV}: \quad 1.337 \mathrm{MV}: 2.176$

$50 \%$ decrease in $\mathrm{T}_{\max }(30 \mathrm{kPa}) \quad \mathrm{AV}: \quad 1.021 \quad \mathrm{MV}: \quad 1.841$

AV: aortic valve, MV: mitral valve.

these two disease features, we observed that aortic velocity could be elevated to $2-3 \mathrm{~m} / \mathrm{s}$, which was similar to that in our cohort of fetal hearts with critical AS. However, interestingly, the MR velocity could not be elevated above $3 \mathrm{~m} / \mathrm{s}$, while many cases in our cohort of fetuses with critical AS had velocities of above $3.5 \mathrm{~m} / \mathrm{s}$. We found that LV wall thickening was necessary to increase $\mathrm{LV}$ pressures further for MR velocities to be elevated to this level, suggesting that such clinical cases might have hypertrophy.

Our simulations further showed AS decreases stroke volume and LV strains more readily than it decreased aortic flow velocity. In our most severe AS cases, stroke volume and strains were close to zero, but aortic velocities remained above $2 \mathrm{~m} / \mathrm{s}$. From our investigation, we found that further reduction of the aortic velocity was possible with further increase in the aortic resistance, and our simulations could produce aortic velocity that is close to zero if resistance was sufficiently high. From our clinical data samples, there were cases where the aortic velocity were less than $1 \mathrm{~m} /$ $\mathrm{s}$, much lower than the average of the whole cohort.

Another noteworthy finding was that AS caused LV strain to decrease much more in the circumferential direction than in the longitudinal direction. Consequently, while our normal hearts had longitudinal strains that were close to their circumferential strain, which was in agreement with a previous study, ${ }^{23}$ our models predicted that diseased hearts had different longitudinal and circumferential strains. Fetal myocardium speckle tracking strain measurements from the literature, however, did not detect such a disparity. ${ }^{24}$ We propose that this mismatch between our simulations and clinical observations can be explained by stenotic fetal heart remodelling its myofiber orientations in a way that reduces the disparity between longitudinal and circumferential strains. We checked the feasibility of this notion with simulations that altered the fiber orientation and found that we could obtain a wide range of differences between longitudinal and circumferential strain. By manipulating fiber angles, strain in a particular direction could switch from positive (stretch) to negative (contract) values (supplementary Fig. S6).

Further, our simulations showed that they could capture the increase in the systole duration and decrease in the diastole duration observed in clinical AS cases. This was observed even without modulating the myocardial activation timing in our model. This phenomenon could be explained by the higher pressure in the LV associated with the disease, which required more time to decrease (via myocardium deformations) to a sufficiently low pressure for mitral inflow to occur.

There are two types of hypertrophy which is possible in the fetal heart, eccentric and concentric hypertrophy. Concentric hypertrophy is typically found in pressure overload situations, such as during fetal AS, while eccentric hypertrophy can be found in fetal growth restriction cases. ${ }^{5}$ In our study, we have modelled only concentric hypertrophy. An increase in LV wall thickness was found to have a significant effect on the hemodynamics and LV function. Changes in the wall thickness substantially enhanced the LV pressure, decreased the stress (myofiber direction) and increased stroke volume. This result is clinically relevant, since hypertrophy was observed in clinical cases of fetal $\mathrm{AS},{ }^{31}$ animal model of aortic banding, ${ }^{10}$ and the KUH clinical data set. It is unclear if this wall thickening is due to cell proliferation or cell enlargement, given that the fetal heart remodels differently from the adult heart. ${ }^{13}$ Our results, however, show that at the average level of hypertrophy of approximately $100 \%$ increase in wall thickness, LV pressures and valve velocities were much higher the average of these parameters in fetal AS patients, suggesting that reduced myocardial contractility is likely to have accompanied hypertrophied AS fetal hearts.

Reduced LV contractility substantially was observed to decrease LV pressure, strain and stroke volume. Contractility may be an important factor influencing the outcomes of fetal aortic valvuloplasty intervention in critical AS cases. Ishii et al. ${ }^{24}$ reported that fetal hearts with AS that demonstrated stronger contractions after aortic valvuloplasty tended to benefit from the intervention and achieve biventricular cardiac function at birth. Conversely, cases that demonstrated lower contractions commonly ended up with univentricular function at birth. With our imagebased FE modeling approach, a potential future 
application is computational estimation of cardiac contractility for prognostication and to predict intervention outcomes.

Interestingly, we found that an increase in myocardial passive stiffness only had very modest effects on myocardial strains and stresses, stroke volume and pressure. This result suggests that endocardial fibroelastosis would not have a major effect on the cardiac function and might not be causing adverse outcomes. Past investigations, however, showed that the severity of fetal LV endocardial fibroelastosis were correlated to the abnormal LV globular geometry, reduced LV function, and lower probability of a biventricular birth outcome. ${ }^{32}$ We suggest that fibroelastosis may simply be a by-product of the disease, rather than being a factor impeding cardiac function to cause poor outcomes.

Compared to adult AS, fetal AS has some similarities and differences. In both scenarios, there are elevated LV pressures, which leads to hypertrophy. ${ }^{3,6}$ However, endocardial fibroelastosis is observed only in fetal $\mathrm{AS},{ }^{32}$ but not in adult AS. Further, the fetal heart undergoes remodeling and morphological changes, and can potentially develop into HLHS, ${ }^{30}$ but the adult heart only hypertrophies. In the fetal heart, it could further be possible for stenosis to be extremely severe, since the right ventricle can sustain circulation. Such a scenario will be fatal in adults, however. Much work has been done for adult FE modelling for pressure overloaded hearts ${ }^{37}$ including modelling of growth and remodelling. ${ }^{39}$ Such work can be very useful if applied to the fetal heart, potentially predicting morphological changes subsequent to disease biomechanics.

There are some limitations associated with this work. Firstly, the small sample size of fetal heart cases used for simulations is a limitation. Secondly, our model omitted a description of the right ventricle, which might affect the spatial distribution of stresses. Thirdly, there could be many concurrent changes during fetal AS development, such as fiber orientation remodelling and changes to myocardial active tension, which were not considered here. Fourthly, our Windkessel model was a simplified representation that did not fully capture fine details of the fetal circulation, and further work to develop a more specific model is underway. Despite these limitations, we believe that the FE model is still useful in developing an understanding of the biomechanical effects of various disease features, and the general trends of these results are unlikely to be affected by these limitations. Finally, our process of optimizing parameters that enables a good geometric fit between the FE model and segmentations from images was conducted manually, and an automated algorithm is likely to further improve the fit.
In conclusion, we conducted a clinical image-based FE modelling of the human fetal heart and demonstrated that it can successfully model normal heart biomechanics as well as the effects of various disease features, such as AS, MR, LV hypertrophy, reduced contractility, and LV fibrosis. AS was found to elevate LV pressures and stress (myofiber direction), and reduce myocardial strains and stroke volume. MR moderates these effects. LV hypertrophy significantly increased LV pressures, strains, and stroke volume and was found necessary to produce high MR valve velocities as measured in the clinic. Reduced contractility significantly decreased LV function, but increased passive myocardial stiffness had only modest effect on LV function and its biomechanical environment.

\section{ELECTRONIC SUPPLEMENTARY MATERIAL}

The online version of this article (https://doi.org/10. 1007/s10439-020-02683-x) contains supplementary material, which is available to authorized users.

\section{ACKNOWLEDGMENT}

This project is financially supported by Singapore Ministry of Education Tier 2 Grant (MOE2018-T2-1003), and Imperial College startup funding. We thank Felicia Toh and Harshaana Ramlal for coordinating the clinical data collection.

\section{CONFLICT OF INTEREST}

All authors have no conflict of interest to declare

\section{OPEN ACCESS}

This article is licensed under a Creative Commons Attribution 4.0 International License, which permits use, sharing, adaptation, distribution and reproduction in any medium or format, as long as you give appropriate credit to the original author(s) and the source, provide a link to the Creative Commons licence, and indicate if changes were made. The images or other third party material in this article are included in the article's Creative Commons licence, unless indicated otherwise in a credit line to the material. If material is not included in the article's Creative Commons licence and your intended use is not permitted by statutory regulation or exceeds the permitted use, you will need to obtain permission directly from the copyright holder. To view a copy of this licence, visit http://crea tivecommons.org/licenses/by/4.0/. 


\section{REFERENCES}

${ }^{1}$ Anthony, J., A. Osman, and M. U. Sani. Valvular heart disease in pregnancy. Cardiovas. J. Afr. 27:111-118, 2016. ${ }^{2}$ Bahlmann, F., S. Wellek, I. Reinhardt, F. Krummenauer, E. Merz, and C. Welter. Reference values of fetal aortic flow velocity waveforms and associated intra-observer reliability in normal pregnancies. Ultrasound Obstet. Gynecol. 17:42-49, 2001.

${ }^{3}$ Chambers, J. The left ventricle in aortic stenosis: evidence for the use of ACE inhibitors. Heart 92:420-423, 2006

${ }^{4}$ Chernyavsky, I. L., O. E. Jensen, and L. Leach. A mathematical model of intervillous blood flow in the human placentone. Placenta 31:44-52, 2010.

${ }^{5}$ Crispi, F., Á. Sepúlveda-Martínez, F. Crovetto, O. Gómez, B. Bijnens, and E. Gratacós. Main patterns of fetal cardiac remodeling. Fetal Diagn. Ther. 47:337-344, 2020.

${ }^{6}$ Davey, B., A. Szwast, and J. Rychik. Diagnosis and management of heart failure in the fetus. Minerva Pediatr. 64:471, 2012.

${ }^{7}$ Dewan S., A. Krishnamurthy, D. Kole, G. Conca, R. Kerckhoffs, M. D. Puchalski, J. H. Omens, H. Sun, V. Nigam and A. D. McCulloch. Model of human fetal growth in hypoplastic left heart syndrome: reduced ventricular growth due to decreased ventricular filling and altered shape. Front. Pediatr. 5: 2017.

${ }^{8}$ Donofrio, M. T., A. J. Moon-Grady, L. K. Hornberger, J. A. Copel, M. S. Sklansky, A. Abuhamad, B. F. Cuneo, J. C. Huhta, R. A. Jonas, and A. Krishnan. Diagnosis and treatment of fetal cardiac disease: a scientific statement from the American Heart Association. Circulation 129:2183-2242, 2014.

${ }^{9}$ Eckersley, L., and L. K. Hornberger. Cardiac function and dysfunction in the fetus. Echocardiography 34:1776-1787, 2017.

${ }^{10}$ Eghtesady, P., E. Michelfelder, M. Altaye, E. Ballard, R. Hirsh, and R. H. Beekman, III. Revisiting animal models of aortic stenosis in the early gestation fetus. Ann. Thorac. Surg. 83:631-639, 2007.

${ }^{11}$ Emmanuel, Y., and S. Thorne. Heart disease in pregnancy. Best Pract. Res. Clin. Obstet. Gynaecol. 29:579-597, 2015.

${ }^{12}$ Essien, S., A. Archibong-Eso, and L. Lao. Discharge coefficient of high viscosity liquids through nozzles. Exp. Thermal Fluid Sci. 103:1-8, 2019.

${ }^{13}$ Foglia, M. J., and K. D. Poss. Building and re-building the heart by cardiomyocyte proliferation. Development 143:729-740, 2016.

${ }^{14}$ Freud, L. R., A. Moon-Grady, M. C. Escobar-Diaz, N. L. Gotteiner, L. T. Young, D. B. McElhinney, and W. Tworetzky. Low rate of prenatal diagnosis among neonates with critical aortic stenosis: insight into the natural history in utero. Ultrasound Obstet. Gynecol. 45:326-332, 2015.

${ }^{15}$ Friedman, W. F. The intrinsic physiologic properties of the developing heart. Prog. Cardiovasc. Dis. 15:87-111, 1972.

${ }^{16}$ Friedman, K. G., L. A. Sleeper, L. R. Freud, A. C. Marshall, M. E. Godfrey, M. Drogosz, T. Lafranchi, C. B. Benson, L. E. Wilkins-Haug, and W. Tworetzky. Improved technical success, postnatal outcome and refined predictors of outcome for fetal aortic valvuloplasty. Ultrasound $\mathrm{Ob}$ stet. Gynecol. 52:212-220, 2018.

${ }^{17}$ Galindo, A., E. Gómez-Montes, O. Gómez, M. Bennasar, F. Crispi, I. Herraiz, A. Mendoza, D. Escribano, E. GarcíaTorres, and J. M. Carretero. Fetal aortic valvuloplasty: Experience and results of two tertiary centers in Spain. Fetal Diagn. Ther. 42:262-270, 2017.
${ }^{18}$ Garcia-Canadilla P., H. Dejea, A. Bonnin, V. Balicevic, S. Loncaric, C. Zhang, C. Butakoff, J. Aguado-Sierra, M. Vázquez and L. H. Jackson. Complex congenital heart disease associated with disordered myocardial architecture in a midtrimester human fetus. Circulation 11: e007753, 2018.

${ }^{19}$ García-Otero, L., O. Gómez, M. Rodriguez-López, X. Torres, I. Soveral, Á. Sepúlveda-Martínez, L. Guirado, B. Valenzuela-Alcaraz, M. López, and J. M. Martínez. Nomograms of Fetal Cardiac Dimensions at 18-41 Weeks of Gestation. Fetal Diagn. Ther. 47:373-384, 2020.

${ }^{20}$ Gardiner, H., A. Kovacevic, G. Tulzer, T. Sarkola, U. Herberg, J. Dangel, A. Öhman, J. Bartrons, J. Carvalho, and H. Jicinska. Natural history of 107 cases of fetal aortic stenosis from a European multicenter retrospective study. Ultrasound Obstet. Gynecol. 48:373-381, 2016.

${ }^{21}$ Guccione, J. M., A. D. McCulloch, and L. Waldman. Passive material properties of intact ventricular myocardium determined from a cylindrical model. J. Biomech. Eng. 113(1):42-55, 1991.

${ }^{22}$ Guccione J. and A. McCulloch. Mechanics of active contraction in cardiac muscle: part I-constitutive relations for fiber stress that describe deactivation. J. Biomech. Eng. 1993; 115(1):72-81.

${ }^{23}$ Ishii, T., D. B. McElhinney, D. M. Harrild, E. N. Marcus, D. J. Sahn, U. Truong, and W. Tworetzky. Circumferential and longitudinal ventricular strain in the normal human fetus. J. Am. Soc. Echocardiogr. 25:105-111, 2012.

${ }^{24}$ Ishii, T., D. B. McElhinney, D. M. Harrild, E. N. Marcus, D. J. Sahn, U. Truong, and W. Tworetzky. Ventricular strain in fetuses with aortic stenosis and evolving hypoplastic left heart syndrome before and after prenatal aortic valvuloplasty. Fetal Diagn. Ther. 35:18-26, 2014.

${ }^{25}$ Johnson, P., D. J. Maxwell, M. J. Tynan, and L. D. Allan. Intracardiac pressures in the human fetus. Heart 84:59-63, 2000 .

${ }^{26}$ Kulkarni, R. P. Surface Motion Analysis for the Natural Ankle and for Total Ankle Replacements with Different Articular Surface Geometries. Philadelphia: Drexel University, 2016.

${ }^{27}$ Lahmers, S., Y. Wu, R. CallDouglas, S. Labeit, and H. Granzier. Developmental control of titin isoform expression and passive stiffness in fetal and neonatal myocardium. Circ. Res. 94:505-513, 2004.

${ }^{28}$ Logg, A., K.-A. Mardal, and G. Wells. Automated Solution of Differential Equations by the Finite Element Method: The FEniCS book. New York: Springer, 2012.

${ }^{29}$ Luewan, S., Y. Yanase, F. Tongprasert, K. Srisupundit, and T. Tongsong. Fetal cardiac dimensions at 14-40 weeks' gestation obtained using cardio-STIC-M. Ultrasound $\mathrm{Ob}$ stet. Gynecol. 37:416-422, 2011.

${ }^{30}$ Makikallio K., J. Levine, G. Marx, S. Colan, A. Marshall, J. Lock and W. Tworetzky. Fetal aortic valve stenosis and the evolution of hypoplastic left heart syndrome: patient selection for fetal intervention. Circulation 110: III690, 2004.

${ }^{31} \mathrm{McCaffrey,} \mathrm{F}$, and F. Sherman. Prenatal diagnosis of severe aortic stenosis. Pediatr. Cardiol. 18:276-281, 1997.

${ }^{32}$ McElhinney D., M. Dr. Vogel, C. Benson, A. Marshall, L. Wilkins-Haug, V. Silva and W. Tworetzky. Assessment of left ventricular endocardial fibroelastosis in fetuses with aortic stenosis and evolving hypoplastic left heart syndrome. The American journal of cardiology 106: 1792-1797, 2010 . 
${ }^{33}$ McPherson, R. A., M. F. Kramer, J. W. Covell, and W. F. Friedman. A comparison of the active stiffness of fetal and adult cardiac muscle. Pediatr. Res. 10:660-664, 1976.

${ }^{34}$ Mekkaoui, C., P. Porayette, M. P. Jackowski, W. J. Kostis, G. Dai, S. Sanders, and D. E. Sosnovik. Diffusion MRI tractography of the developing human fetal heart. PLoS ONE 8:e72795, 2013.

${ }^{35}$ Mesa, A., C. Jessurun, A. Hernandez, K. Adam, D. Brown, K. Vaughn William, and S. Wilansky. Left ventricular diastolic function in normal human pregnancy. Circulation 99:511-517, 1999.

${ }^{36}$ Mirbod, P. Analytical model of the feto-placental vascular system: consideration of placental oxygen transport. $R$. Soc. Open Sci. 5:180219, 2018.

${ }^{37}$ Mojumder J., J. Choy, S. Leng, L. Zhong, G. Kassab and L. Lee. Mechanical stimuli for left ventricular growth during pressure overload. Exp. Mech. 1-16, 2020.

${ }^{38}$ Nakayama Y. Action of the fluid in the air-micrometer: $1 \mathrm{st}$ report, characteristics of small-diameter nozzle and orifice no. 1, in the case of compressibility being ignored. Bull. JSME 4: 507-515, 1961.

${ }^{39}$ Niestrawska, J. A., C. M. Augustin, and G. Plank. Computational modeling of cardiac growth and remodeling in pressure overloaded hearts-Linking microstructure to organ phenotype. Acta Biomater. 106:34-53, 2020.

${ }^{40}$ Ohayon, J., Y. Usson, P.-S. Jouk, and H. Cai. Fibre orientation in human fetal heart and ventricular mechanics: a small perturbation. Comput. Methods Biomech. Biomed. Eng. 2:83-105, 1999.

${ }^{41}$ Piroddi, N., A. Belus, B. Scellini, C. Tesi, G. Giunti, E. Cerbai, A. Mugelli, and C. Poggesi. Tension generation and relaxation in single myofibrils from human atrial and ventricular myocardium. Pflügers Archiv 454:63-73, 2007.

${ }^{42}$ Racca, A. W., J. M. Klaiman, J. M. Pioner, Y. Cheng, A. E. Beck, F. Moussavi-Harami, M. J. Bamshad, and M. Regnier. Contractile properties of developing human fetal cardiac muscle. J. Physiol. 594:437-452, 2016.

${ }^{43}$ Rugonyi, S. Genetic and flow anomalies in congenital heart disease. AIMS Genet. 3:157-166, 2016.

${ }^{44}$ Sahasakul, Y., W. D. Edwards, J. M. Naessens, and A. J. Tajik. Age-related changes in aortic and mitral valve thickness: implications for two-dimensional echocardiography based on an autopsy study of 200 normal human hearts. Am. J. Cardiol. 62:424-430, 1988.
${ }^{45}$ Shavik, S. M., Z. Jiang, S. Baek, and L. C. Lee. High spatial resolution multi-organ finite element modeling of ventricular-arterial coupling. Front. Physiol. 9:119, 2018.

${ }^{46}$ Shavik, S. M., S. T. Wall, J. Sundnes, D. Burkhoff, and L. C. Lee. Organ-level validation of a cross-bridge cycling descriptor in a left ventricular finite element model: effects of ventricular loading on myocardial strains. Physiol. Rep. 5:e13392, 2017.

${ }^{47}$ Shuffrey, L. C., M. M. Myers, H. J. Odendaal, A. J. Elliott, C. du Plessis, C. Groenewald, L. Burd, J. Angal, J. D. Nugent, and J. R. Isler. Fetal heart rate, heart rate variability, and heart rate/movement coupling in the Safe Passage Study. J. Perinatol. 39:608-618, 2019.

${ }^{48}$ Uittenbogaard, L. B., M. C. Haak, M. D. Spreeuwenberg, and J. M. van Vugt. Fetal cardiac function assessed with four-dimensional ultrasound imaging using spatiotemporal image correlation. Ultrasound Obstet. Gynecol. 33:272-281, 2009.

${ }^{49}$ Wenk, J. F., D. Klepach, L. C. Lee, Z. Zhang, L. Ge, E. E. Tseng, A. Martin, S. Kozerke, J. H. Gorman, 3rd, R. C. Gorman, and J. M. Guccione. First evidence of depressed contractility in the border zone of a human myocardial infarction. Ann. Thorac. Surg. 93:1188-1193, 2012.

${ }^{50}$ Wiputra, H. Flow dynamics in healthy and tetralogy of fallot fetal ventricles. Singapore: National University Of Singapore, 2019.

${ }^{51}$ Wiputra, H., C. K. Chen, E. Talbi, G. L. Lim, S. M. Soomar, A. Biswas, C. N. Z. Mattar, D. Bark, H. L. Leo, and C. H. Yap. Human fetal hearts with tetralogy of Fallot have altered fluid dynamics and forces. Am. J. Physiol. 315:H1649-H1659, 2018.

${ }^{52}$ Wiputra H., G. L. Lim, D. A. K. Chia, C. N. Z. Mattar, A. Biswas and C. H. Yap. Methods for fluid dynamics simulations of human fetal cardiac chambers based on patientspecific 4D ultrasound scans. J. Biomecha. Sci. Eng. 11: 1500608-00615-00608, 2016.

${ }^{53}$ Zampi J. D., M. C. Treadwell, C. G. Fifer, C. van de Ven, A. P. Rocchini, W. Whiteside and S. K. Gelehrter. Intracardiac hemodynamics during fetal balloon aortic valvuloplasty. JACC: Cardiovas. Interv. 12: 1628-1630, 2019.

Publisher's Note Springer Nature remains neutral with regard to jurisdictional claims in published maps and institutional affiliations. 